\title{
Zwei cromerzeitliche Artefakt-Fundplätze in der Jüngeren Hauptterrasse am Niederrhein
}

\author{
KLAUS Schmude *)
}

Lower Pleistocene, Rhine River, Younger Main Terrace, Matuyama-Brunhes-Limit, gravel-pits, artefacts, quartzit/quartzitic sandstone, quartz, flint.

Lower Rhine Embayment

Kurzfassung: In zwei aus den Flußterrassen des Rheines fördernden Sand- und Kiesgruben fanden sich Stein-Artefakte, in der Mehrzahl abgerollt: sie sind überwiegend aus Varianten von Quarzit und quarzitischem Sandstein hergestellt, einige aus Quarz oder Flint.

Die Betriebe, Weeze und Kirchhellen, fördern Sand und Kies aus der Hauptterasse 3 im ersten und der Hauptterrasse 1 im zweiten Fall. Die paläomagnetische Matuyama-Brunhes-Grenze befindet sich im oberen Teil der Hauptterrasse 3. Daher sind Sedimente und Artefakte in beiden Kiesgruben älter als ca. 700000 Jahre.

Die Oberflächen der überwiegend abgerollten und/oder patinierten Artefakte (kein Windschliff) weisen den gleichen Schliff und Färbung wie die Gerölle des Kieses auf. Sowohl auf den Geröllhalden als auch auf den Abbauflächen wurde gesammelt. In der Kiesgrube Kirchhellen wurden zwei Artefakte aus der Schicht geborgen.

Nach den heutigen Vorstellungen fand die Bildung der Jüngeren Hauptterrassen zwischen 0,6 und 1,6 Millionen Jahren statt, je nach Interpretation der paläomagnetischen Meßergebnisse sowie nach der schwermineralanalytischen Verknüpfung der Terrassenkörper.

Das Inventar der Artefakte umfaßt Bifaces, Trieder, Pics, Cleaver, Chopper/Chopping Tools, Polyeder, Abschläge und Kerne.

Größere Gerölle sind selten; dies mag die Seltenheit von großen Abschlägen, die in Inventaren des Mittelmeerraumes zur Herstellung von Faustkeilen und Cleavern dienten, erklären. Ein unbekannter Prozentsatz von Quarz- und FlintArtefakten dürfte bis zur Unkenntlichkeit verändert worden sein; dasselbe dürfte auf die Kleingeräte zutreffen.

Bedauerlicherweise gibt es in beiden Kiesgruben keine Faunenreste.

Die Inventare sind selbst mit ihrer begrenzten und durch die Fundbedingungen selektierten Artefaktzahl bemerkenswert. Man kann ihr Vorkommen im gleichen Rahmen wie die Fundstücke der älteren Schichten von Mülheim-Kärlich

*) Anschrift des Verfassers: K. SCHMUdE, Habichtstr. 71, D - 4300 Essen 1. sowie dem Koblenzer Raum sehen. Im Unterschied zu diesen enthalten sie jedoch typische Geräte, welche Aussagen zu ihrer Position in Technik und Zeit erlauben. Sie dürften dem frühen Cromer-Komplex angehören. Der kulturelle Rahmen muß im frühesten Beginn der europäischen Faustkeilkultur, früher auch Abbevillien, heute als Früh-Acheuléen, Alt-Acheuléen oder Proto-Acheuléen bezeichnet, gesehen werden.

[Two Cromerian Artefact Sites in the Younger Main Terrace in the Lower Rhine Area]

Abstract: In two gravel-and-sand-pits, producing from fluviatil terraces of the river Rhine, lithic artefacts were encountered, in the mayority rolled, made mainly on various sorts of quartzite and quartzitic sandstone, a few on quartz and flint.

The pits, Weeze and Kirchhellen, produce sands and gravels from the Younger Main Terrace (HT 3) in the first and Younger Main Terrace (HT 1) in the second case. Consequently sediments and artefacts in these two pits are older than 700000 years.

The surfaces of the mostly rolled and/or patinated artefacts (no eolisation) have the same polish and coloration as the pebbles from the gravel. Collecting took place on the pebble-storage-dumps as well as along the production-front. Two artefacts were taken directly from the sediments in the pit Kirchhellen.

Present geological thinking sees the formation of the Younger Main Terraces from 0,6 to 1,6 millions years, depending on the interpretation of the paleomagnetic measurements and of the aspect of the heavy-minerals-analysis of the terrace-bodies.

The inventory of the artefacts contains bifaces, trihedrals, picks, cleavers, choppers/chopping tools, polyhedrons, flakes and cores. Bigger pebbles are scare: this may explain, why large flakes, which served in inventories of the Mediterranean Area for the production of bifaces and cleavers, are rare. An unknown percentage of quartz- and flint-artefacts was probably deteriorated to the point of being un-identifiable; the sample applies to small (or light-duty-) tools. 
Regrettably there are no rests of fauna in both pits. These inventories are, even with their limited and, by the existing conditions, selected number of artefacts, remarkable. Their presence can be seen in context with the artefact-sites of the elder layers from Mülheim-Kärlich as well as the Koblenzarea. In difference to these they contain typical tools, which permit conclusions regarding their position in technique and time. Apparently they belong into the early Cromercomplex.

The cultural context is to be seen in the very beginning of the european hand-axe-culture, formerly known as Abbevillian, now defined as Early Acheulian, Lower Acheulian or Proto-Acheulian.

\section{Forschungsstand}

Die Erforschung des Altpaläolithikums in Deutschland fand, im Vergleich mit Ländern wie Frankreich und Spanien, nur sporadisch und zögernd statt. Darauf dürften unter anderem folgende Gründe Einfluß gehabt haben:

a) In den oben erwähnten Ländern ist ein außerordentlicher Fundreichtum vorhanden, besonders auf bzw. in den Flußterrassen.

b) Die Diskussion um die Funde und Theorien RusT's (1971), die „Heidelberger Kultur” und deren Ablehnung, zog als Folgeschäden zwei Erscheinungen nach sich:

- eine große Zahl von Sammlern legte der Fachwelt Phantasieprodukte vor,

- als Folge auch davon baute sich bei den deutschen Archäologen gegenüber abgerollten und/oder oberflächenveränderten Fundstücken eine von vornherein ablehnende Haltung auf.

Als weiteres negatives Moment sei hier auch die sog. "faustkeilfreie Zone" genannt, welche den Rhein als Grenze faustkeilführender Industrien postuliert.

Die Mittelpaläolithischen Funde wurden im westlichen Mitteleuropa von BOSINSKI (1967) aufgearbeitet und nach kulturellen, chronologischen und typologischen Gesichtspunkten gegliedert. Für das folgende Altpaläolithikum fehlt jedoch eine solche ausführliche Aufarbeitung. Im Schriftgut anderer Länder sind nur Regionen bearbeitet. In Gesamtübersichten des Paläolithikums wie den Arbeiten von BORDES (1984) oder MÜLLER-KARPE (1966) nimmt das Altpaläolithikum nur einen untergeordneten Raum ein. Lediglich FIEDLER (1985) bietet eine Einführung in die typologischen Probleme des Altpaläolithikums.

Hinzu kommt, daß die Situation in der Quartärgeologie in Fluß geraten ist; neue Erkenntnisse, besonders durch absolute Altersangaben wie z.B. in Mühlheim-Kärlich (SCHMINCKE \& BOOGARD 1989), dazu der Prozeß der Lösung von den klassischen alpinen Stufen und die Versuche der Korrelation mit den Stufen der Tiefsee-Bohrkerne, schaffen Unsicherheit. Erwähnt man noch die Schwierigkeiten bei der Ansprache oberflächenveränderter Artefakte, wird verständlich, daß all diese Umstände keinen Ansporn bilden, sich mit dem genannten Fundstoff zu beschäftigen.

Funde des Jüngeren Acheuléen sind dagegen aus Deutschland reichlich bekannt, so z. B. aus Markkleeberg, dem Leine-Tal, Wattenstedt, dem Emscherraum, die Quarzitschlagstellen von Hessen, um eine Reihe zu nennen.

An älterpaläolithischen Fundstellen sind in der BRD und im Grenzbereich zu nennen:

a) Die Geröllgeräte von Achenheim im Elsaß (THEvENIN 1976)

b) Die Funde van BERG's und FiedLeR's (1983) von Winningen/Bisholder an der Mosel, aber auch schon vorher der Hinweis auf ältere paläolithische Funde am Mittelrhein (FIEDLER 1977).

c) Die von WÜRGES (1986) geborgenen Fundstücke von Mülheim-Kärlich, die in das späte Alt- und/oder das frühe Mittel-Pleistozän zu datieren sind.

d) Die Münzenberger Geröllgeräte (KRÜGER 1980)

e) Der Unterkiefer von Mauer

Die altpaläolithischen Artefakte des Rheinlandes wiesen Merkmale auf, die auch bei der Suche und dem Erkennen derartiger Funde in anderen Zonen dieses Gebietes beachtet werden müssen (FIEDLER 1989):

- die Fundstücke waren oft aus meist grobem, zähen, nur gelegentlich auch feinkörnigerem, spröden Material gefertigt, das für die Erkennbarkeit der technologischen Kennzeichen ihrer anthropogenen Herstellung viel Erfahrung verlangt.

- bis auf die im Löss gefundenen wiesen diese Steingeräte sehr unterschiedliche Erhaltungs- und Verwitterungszustände auf.

- mit Ausnahme der wenigen Faustkeile von Kärlich und Bisholder waren die übrigen Artefakte sehr einfache Chopper, Abschläge und Kerne, deren anthropogener Ursprung im jeweiligen Einzelfall nicht unumstritten zu sein braucht und die typologisch wenig aussagen.

2 Entdeckung, Lage, Geologie und stratigraphische Zuordnung der Fundstellen

1985 begann, unter Zuhilfenahme geologischer Unterlagen, im Raum um Essen die Suche nach paläolithischen Artefakten und zwar in Anlehnung an die allgemeinen Erfahrungen mit den FlußterrassenFundstellen Südfrankreichs und Spaniens. Diese führ- 


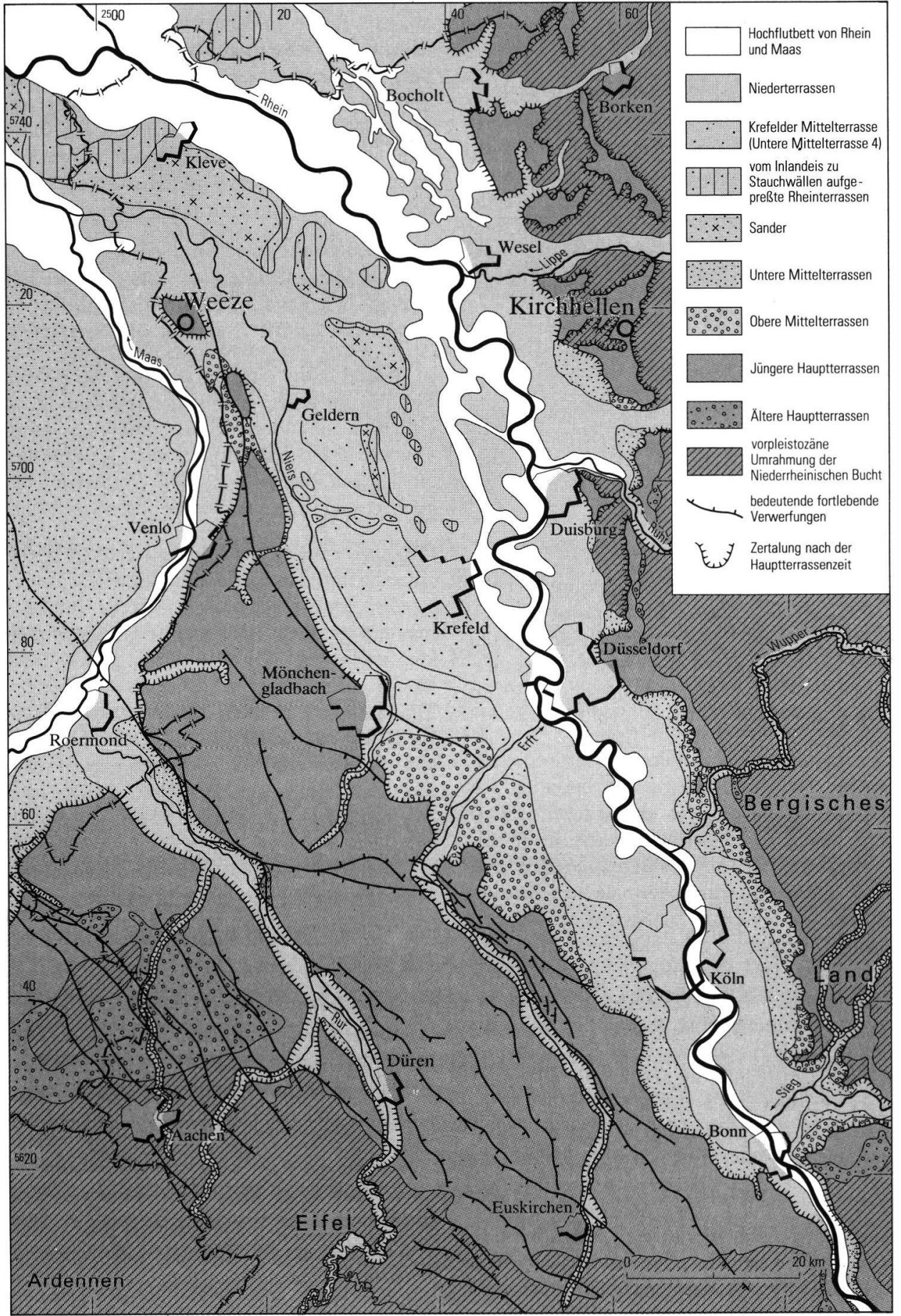

Abb. 1: Lageplan. 
te 1989 zu den beiden Sand- und Kiesgruben Weeze und Kirchhellen, deren Fundsituation und -material im folgenden beschrieben wird.

Die Sand- und Kiesgrube Weeze befindet sich ca. $6 \mathrm{~km}$ westlich dieses Ortes auf einem geologischen Halbhorst. Hier waren die verwilderten AbflußSysteme von Rhein und Maas während der Bildung der Hauptterrassen verzweigt und vernetzt, die Sedimente sind daher eine Mischfazies.

Die Sand- und Kiesgrube Kirchhellen befindet sich ca. $3 \mathrm{~km}$ südwestlich dieses Ortes. Rhein- und RuhrMaterial sind hier gemischt vorhanden. Auffällig ist der hohe Flint-Anteil, z. B. in kopfgroßen Knollen, wohl aus dem Aachener Raum.

$\mathrm{Da}$ beide Fundstellen in Jüngeren Hauptterrassen liegen und zwar in Weeze gesichert (KLOSTERMANN 1984: 492) in der HT 3, Kirchhellen in der HT 1 (BRUNNACKER et al. 1982: 216), sollen sie vorerst gemeinsam behandelt werden.

Die Bildung der Jüngeren Hauptterrassen wird von ca. 0,6 Mio. Jahren bis ca. 1,6 Mio angesetzt (BRUNNACKER 1978). Sie liegt damit zum großen Teil im Cromer-Komplex. Die Grenze zwischen der letzten Periode normaler (Brunhes) und reverser Polarisierung (Matuyama) liegt bei 0,7 Mio. Jahren. Sie verläuft im oberen Drittel der HT 3.

In $\mathrm{W}$ e e $\mathrm{z}$ e wurde reverse Polarisierung gemessen mdl. Mitteilung J. KLOSTERMANN), hier befinden sich also, auch auf Grund anderer geologischer Daten, Schichten des älteren Teiles dieser Terrasse, deren Bildung von ca. 0,6 bis 0,8 Mio. Jahren angesetzt wird. In $\mathrm{K}$ i r c h h e 11 e $\mathrm{n}$ wurde normale Polarisierung gemessen (mdl. Mitteilung F. JANSEN); es handelt sich hierbei um ein vorläufiges Ergebnis; die Bestätigung bleibt abzuwarten. Diese Polarisierung deutet auf das Jaramillo-Ereignis (ca. 0,9-0,95 Mio. J.). Unsere, sowie eine weitere Fundstelle ca. $10 \mathrm{~km}$ nördlich, mit ebenso wahrscheinlich normaler Polarisierung liegen in einem Terrassenstreifen, der sich von Bottrop nach NE erstreckt und von BRUNNACKER et al. (1982: 216) in die HT 1 eingestuft wurde. Das Alter der Bildung dieses Terrassenkomplexes wird von ca. 0,85 bis 1,65 Mio J. angesetzt. Das Jaramillo-Ereignis liegt im jüngsten Teil dieser Perioden. Erwähnenswert erscheint, daß in Mülheim-Kärlich die ältesten Artefaktfunde ebenfalls aus diesem Bereich stammen. Das Alter der Artefakte wird auf ca. 1 Mio J. geschätzt (WÜRGES 1986: 3)

In beiden Kiesgruben setzen sich die Schichten aus häufig wechselnden bräunlich- oder auch schwarz gefärbten Sand-, Kies- und Tonlagen zusammen. Die Mächtigkeit der Terrassen liegt zwischen 4 und $8 \mathrm{~m}$. Die Sand- und Kieslagen schwanken in ihrer Ausbil- dung, Ton- oder Schlufflagen schieben sich linsenförmig ein, Rinnenbildung und Schräg- bzw. Diagonalschichtung wechseln auf kurzer Distanz: das typische Bild eines verwilderten Flusses mit stark schwankender Wasserführung; die Artefakte sind daher sekundär eingespült. Geologische Anzeichen für die Einordnung in die Hauptterrasse 3 sind u. a. vom Eis mitgenommene Tongerölle und synsedimentäre Eiskeile, beide Merkmale sind für diese Stufe typisch.

In beiden Gruben werden die Überkornhalden, aber auch die Abbaufronten abgesucht. In der folgenden geologischen Beschreibung wird begründet, daß die Fundstücke aus den Terrassen selbst stammen.

In Weeze bildet die Terrasse die obere Schicht des Profiles, sie reicht bis in die Ackerkrume. Darüber gibt es keine andere Schicht. Die oberste Partie der Terrasse unter dem Humus weist gelegentlich Züge einer alten Oberfläche auf. Die Gerölle sind bräunlich verfärbt und tragen teilweise Spuren von Windschliff.

Die obere, etwa $2 \mathrm{~m}$ mächtige Schicht der Terrasse wird häufig durch Kryoturbationen gestört. Diese Taschen enthalten lehmiges Material, das mit Terrassengeröll gemischt ist; Gerölle daraus sind meist dunkelbraun gefärbt. Die Kryoturbationen wurden mehrfach gründlich untersucht, jedoch keine Artefakte gefunden. Solche müßten deutlich dunkler gefärbt sein als die Funde aus der Terrasse. Gerölle und Artefakte aus der Terrasse sind weiß bis gelb gefärbt. Die Abrollung von Geröllen und Artefakten ist gleichartig. Die Negative an den Artefakten weisen fast dieselbe Patina oder Verfärbung auf wie die Terrassengerölle. Bisher konnte, im Gegensatz zu Kirchhellen, noch kein Stück aus der Schicht geborgen werden.

Die Terrassenschichten werden von pliozänen Sanden und Tonen unterlagert.

Geologische Schicht-Profile werden nicht im Detail beschrieben. Bei dem schnellen Wechsel auf oft kurzer Distanz sagt ein Einzel-Profil nichts aus. Es lassen sich jedoch einige Regelmäßigkeiten an beiden Profilen erkennen: die größere, untere Partie besteht aus einem Sand-Kies-Gemisch, mäßig durchsetzt mit Geröllen. Darauf liegt eine Geröllbank von 0,5 bis 1,0 , gelegentlich 2,0 m Mächtigkeit; aus dieser konnten in Kirchhellen zwei Fundstücke geborgen werden. In Weeze stammen die Funde wohl ebenfalls aus dieser Geröllbank.

Darüber liegt in beiden Gruben ein Sand-Kies-Gemisch, welches durch Frost gestört ist.

In Kirchhellen liegt am Top eine 1-2m mächtige wohl drenthezeitliche Grundmoräne. Die Gerölle der Moräne bestehen überwiegend aus mitgeschlepptem Material der Terrasse, nordische Geschiebe und Flint 
sind selten. Unter der Moräne lagern, wie schon erwähnt, Sande und Kiese der Hauptterrasse, die der HT 1 angehören.

Unter diesen Schichten befinden sich oligozäne Tone. Die Abrollung von Geröllen und Artefakten ist gleichartig, ebenso der Oberflächenzustand sowie die hell- bis dunkelgelbliche Verfärbung. Darüber hinaus sind einige Artefakte (z.B. Kihe 144), teilweise in typischem Mangan-Schwarz gefärbt, ein Hinweis auf die Einbettung des betreffenden Stückes in eine der entsprechenden Zonen der Terrasse. Wie erwähnt, wurden 2 Fundstücke aus der Schicht, der Geröllbank hier, geborgen (Kihe 5 und Kihe 193).

Im folgenden wird der Zustand der als Rohmaterial verwendeten Gerölle beschrieben. Das durch Sieben ausgesonderte Überkorn enthält mehr oder weniger, in der Mehrzahl erheblich verrundete Gerölle. Der Anteil an fast völlig runden oder ovalen Stücken ist jedoch gering. Die Mehrzahl der Gerölle ist patiniert, ein Teil in hohem Maß. Schlägt man solche Stücke an, erkennt man häufig die mehrere mm starke Kruste; das Innere ist meist mürbe, ausgelaugt und fast immer heller gefärbt als die Kruste. Neben den gerundeten Geröllen kommen nicht selten etwa 15 bis $30 \mathrm{~cm}$ lange, nicht so stark verschliffene Frostscherben vor, die im Vergleich zu den übrigen Geröllen weit besser enthalten sind (kurzer Weg/Transport im Eis/Zerfall im Frost auf einer Kiesbank?). Die Rheingerölle werden in der allgemeinen geologischen Literatur, z. B. den Erläuterungen zum Blatt Geldern (KLOSTERMANN 1984) ausführlich beschrieben.

\section{Das Fundmaterial}

\subsection{Das Rohmaterial}

Im folgenden werden die Gesteine beschrieben, aus denen Artefakte geschlagen wurden, dazu ihre Verwendbarkeit, Unterschiede in derselben und spätere Veränderungen. Den Hauptanteil bilden Varianten aus einer Reihe „Sandstein - quarzitischer Sandstein - Quarzit". Aus anderem Material wurden nur vereinzelt Artefakte gefunden, doch dürften Probleme in der Ansprache von z. B. Quarz oder Flint dafür verantwortlich sein.

Aus folgenden Gesteinen wurden Artefakte hergestellt:

a) T a u nus qu a r zi t : dieser umfaßt Quarzitgerölle aus dem Hunsrück-Taunus-Bereich: meist grober, sehr unterschiedlicher Quarzit, manchmal fast Sandstein, daneben glasige Varianten, von bläulicher, rötlicher, violetter, auch weiß-grau bis gelblicher Färbung. Die Schlagmarken sind häufig gut ausgeprägt mit Bulbus, Auftreffpunkt und Radialstrahlen. Häufig vorhandenes Gestein, häufig verwendet. b) Quarzite und quarzitische Sand. steine aus Devon/Karbon: Es kommen alle Varianten vor. In frischem Zustand besitzt dieses Gestein oft einen typischen stahlgrauen Farbton. Wenn verwittert, wird es gelblich-grün bis olivgrün. Schlagmarken sind nur gelegentlich gut zu erkennen. Die durch das Zusammentreffen von Negativen gebildeten Grate sind typisch gerade, nicht geschwungen, wie meist bei den anderen $\mathrm{Ge}$ steinen. In Kirchhellen häufig (Ruhr-Anteil), in Weeze sehr selten.

c) P y r i t q u a r z i t : meist feinkörniger, oft schiefriger Quarzit von grau-schwarzer Färbung, durch viele Löcher ausgewitterter Pyritkristalle gekennzeichnet. Erfordert hohen Kraftaufwand beim Bearbeiten; massive Stücke stehen bei Beanspruchung lange.

d) Spröder Tertiärquarzit: dieser und der folgende Quarzit sind Varianten des äußerst unterschiedlich vorkommenden Tertiärquarzites. Diese gelegentlich verwendete Form ist feinkörnig, guterhalten fast glasig. Ausgelaugt erscheinen die Stücke fast mürbe, die Kanten sind stark verrundet. Er ist immer gelblich-hellbraun gefärbt. An besser erhaltenen Stücken sind Schlagmarken gut zu erkennen. Dieser Quarzit wurde im Jung-Acheuléen häufig verwendet.

e) Zäh e r T e r t i är qu a r z i t : sehr feinkörniger, dichter, zäher Quarzit, meist gelblich-bräunlich, aber auch grau und grünlich gefärbt. Enthält häufiger Versteinerungen. Er bildet eine auffällige prozellanartige, fleckige, weißlich-gelbe Patina. Oft von schiefriger Struktur, springt unregelmäßig und bildet auffällig irreguläre Formen. Schlagmerkmale häufig unklar, sind jedoch gut zu erkennen, wenn man selbst schlägt und entsprechend Erfahrung sammelt. Bildet scharfe Kanten, die unter schwerer Beanspruchung lange stehen. Schwer bearbeitbar, ist er jedoch der widerstandsfähigste der vorkommenden Werkstoffe.

f) Q u a r z : es wurden vereinzelt Kerne und Geräte gefunden. Ein Erkennen ist nur möglich, wenn neben halbwegs gut erhaltenen Schlagmerkmalen auch eine deutliche Funktionsform erkennbar ist.

g) F l i n t: in Weeze wurden drei Abschläge gefunden. Schwerpunkte bei der Artefaktherstellung scheinen beim Taunusquarzit und dem zähen Tertiärquarzit zu liegen. Diese Aussage ist mit Vorsicht zu betrachten, da die Fundumstände die Verhältnissse verfälschen dürften.

\subsection{Erhaltungszustand der Artefakte}

Die Artefakte weisen alle möglichen Abrollungs-Stadien auf, gesteinsbedingte Unterschiede sind nicht zu erkennen. Von Taunusquarzit und zähem Tertiärquarzit liegen einige besser erhaltene Fundstücke vor. Die Mehrzahl der Fundstücke ist stark bis sehr stark verrollt. Die Fundzahl ist zu gering, um zu versuchen, einen Maßstab für Abrollung aufzustellen. So lassen sich bis jetzt keine Aussagen machen, wie sie z. B. in Pinedo möglich sind (QUEROL \& SANTONJA 1979: 45). Eine kleine Zahl Artefakte ist stark patiniert. Windschliff ist nicht zu erkennen, wohl nicht vorhanden. 


\subsection{Schlagtechnik und andere technologische Aspekte}

Die verwendeten Rohstoffe sind im Verhalten und der Nutzung sehr unterschiedlich. Bei Schlagversuchen wurde folgende Beobachtung gemacht:

der Werkstoff mit der größten Standfestigkeit ist der zähe Tertiärquarzit, seine Schneiden überstehen Bearbeitung von hartem Holz und Hirschgeweih weit besser als die übrigen Rohstoffe. Seine Bearbeitung erfordert ungewöhnilchen Krauftaufwand; die massivere Form des Pyritquarzites kommt ihm nahe.

Der Taunusquarzit ist am besten von allen Rohmaterialien bearbeitbar, seine Arbeitskanten verstumpfen aber schneller. Die restlichen Gesteine fallen gegen die vorher genannnten $a b$.

Der Artefaktanteil an Flint ist viel geringer als der Anteil von Flintknollen und -brocken im Geröll der Terrasse. Man sollte eigentlich sowohl größere, vor allem aber kleinere Flintartefakte erwarten.

Wie auf anderen altpaläolithischen Fundstellen dürften jedoch teilweise die Grobgeräte aus anderen, für die schweren Hack-Arbeiten besser als Flint geeigneten Gesteinen wie z. B. Quarzite, geschlagen worden sein, während für die feineren Arbeiten Geräte aus Flint verwendet wurden. Diese hypothetischen Kleingeräte dürften vom Fluß zerstört oder weggespült worden sein.

Die gefundenen Artefakte sind überwiegend mit wenigen Schlägen geformt, wozu ganz erhebliche Kraft notwendig ist. Daneben sind aber, z. B. an Triedern, Flächen mit vielen feineren Schlägen bearbeitet. Geschlagen wurde, so zum Beginn eines Gerätes, wohl auf dem ruhenden Amboß, aber auch ungewöhnlich oft mit bipolarer Technik. Die Kanten sind teils stark sinusförmig gewellt, einige Stücke haben aber bereits recht gerade Kanten.

Die Geräte sind meist sehr dick, oft unsymmetrisch und irregulär. Auffällig ist das seltene Vorkommen großer Abschläge, was mit der Seltenheit großer Gerölle zusammenhängen dürfte. Plattiges Material kann als Ersatz für große Abschläge gedient haben.

\subsection{Formenkundliche Ordnung}

Mit dem Auffinden der Artefakte wurde es notwendig, nach vergleichbaren Typen zu suchen, da solche in den Beschreibungen nord- und mittel-europäischer Fundstellen nicht vorkommen und damit Beschreibungen fehlen, die zu Vergleichszwecken herangezogen werden könnten. Solche finden sich jedoch in Inventaren bekannter Alt-Acheuléen-Fundstellen des Mittelmeer-Raumes und werden hier verwendet. Dabei wurde besonders auf die von TAvoso (1978) und
SANTONJA (QuEROL \& SANTONJA 1979) beschriebenen Typen zurückgegriffen.

\section{Faustkeile}

Eine Reihe von Autoren, die sich mit Alt-AcheuléenInventaren befaßt haben, bezeichnen als Faustkeile alle mit der Faust geführten, keilförmigen bis spitzen Geräte (Bifaces, Pics, Trieder, Cleaver). Diese Betrachtungsweise wird hier auch verwendet, wobei von „Faustkeilen in s.1.” und „Bifaces = Faustkeile in s. s." die Rede ist. Um der besseren Vergleichbarkeit halber werden einzelne Geräte-Typen, soweit möglich, getrennt behandelt. Siehe hierzu auch BORDES (1988: 77) und LEAKEY (1971: 5).

P i c s u r dièd r e c o r t i c a l, nach Tavoso (1978: 28)

Kommen in flacherer Form an der Garonne, im Mittleren Acheuléen vor; in gröberer Form z. B. in Pinedo (QUEROL \& SANTONJA 1979: 124-141)

Terra Amata Pic, mit abrupter R e t u s c h e, nach TAvoso (1978: 28)

Vorkommen im Mittleren und Alt-Acheuléen, nicht häufig, aber fast immer vorhandenes Gerät. (QUEROL \& SANTONJA 1979 124-128, AgUADO 1963: $\mathrm{T}$. 13-28, Lumley 1976; SAntonja \& Perez-Gonzales 1984: 147, 148)

\section{$\mathrm{P}$ i c a l t è $\mathrm{r} \mathrm{n}$ e nach TAvoso (1978: 28)}

An diesem Gerät ist eine Spitze oder kleine Meißelschneide durch alternierendes Beschlagen der Seiten eines flachen Gerölles herausgearbeitet. Das zeitliche Auftreten entspricht dem der vorher genannten Geräte.

Für die Beschreibung der anderen Fundstücke genügen bekannte Begriffe.

Generell soll auf die erhebliche Unsicherheit in der hier verwendeten Klassifizierung hingewiesen werden: wir wissen nicht, welches die reale Funktion der Artefakte war und inwieweit die aus unserer heutigen Sicht herausragenden Formen wirklich das waren, was wir vermuten. Die Unklarheit der Abgrenzung Chopper/Chopping Tool gegen Kerne ist bekannt (TOTH 1987: 109, 110), Abgrenzungen wie Pic-Trieder-Pic sur dièdre cortical - Terra Amata-Pic mit abrupter oder flächiger Retusche - Chopper à pointe sind sicher künstlich und dazu in so hohem Maße vom vorhandenen Material abhängig, daß man sie nur als Versuch der Anwendung unvollkommener Hilfsmittel (mangels Besserem) ansehen sollte. Was „sicher" erscheint, ist, daß der Frühmensch hier anscheinend viele derbe Spitzen herstellte - über die Funktion können wir Vermutungen anstellen. 


\subsection{Gefundene Artefakte}

\subsubsection{Kerne}

Die bisher gefundenen Kerne weisen folgende Merkmale auf:

- sie wurden alle unsystematisch abgebaut,

- nur an zwei Exemplaren ist eine simple Kantenpräparation (?) angedeutet,

- die Zahl der Negative reicht von 2 bis 8,

- an 4 Stücken ist bipolare Technik erkennbar.

(Diese Kerne zeigen auf der Abbaufläche einen Schlagwinkel von ca. $90^{\circ}$, am distalen Kern-Ende eine Zone auffälliger Zertrümmerung (vgl. KoBAYASHI 1975).

- viele Kerne besitzen unregelmäßige, grobe, ovale, bis würfelförmige Körper. Siehe Taf. 4, Fig. 1 und 3, Taf. 5, Fig. 1 und Taf. 6, Fig. 1 a.

Ein Teil der Fundstücke könnte sowohl als „Chopper” als auch als „Kern” bezeichnet werden; es gibt ein Mischfeld. Diese Stücke wurden im Prinzip alle den Kernen zugeordnet.

\subsubsection{Abschläge}

Die Fundstücke sind überwiegend dorsal cortexbedeckt, Negativreste sind dorsal selten. Die Schlagflächenreste sind jedoch überwiegend cortexfrei, also Negativreste. Abschläge wurden aus fast allen vorkommenden Gesteinen hergestellt, lediglich Quarz und Pyritquarzit wurden nicht angetroffen. Diedrische oder facettierte Schlagflächenreste kommen nicht vor.

Die Größe der Fundstücke liegt zwischen ca. $150 \mathrm{~mm}$ Breite, $100 \mathrm{~mm}$ Länge und $60 \mathrm{~mm}$ Dicke sowie etwa $70 \times 60 \times 30 \mathrm{~mm}$ (s. Taf. 4, Fig. 2).

\subsubsection{Bearbeitete Abschläge}

Geräte wie Schaber, Spitzen o. ä. an Abschlägen wurden bisher nicht gefunden.

\subsubsection{Beschlagene Gerölle}

Von den beiden Fundstellen liegen Chopper und Chopping Tools vor. Auffällig ist die geringe Fundzahl an solchen Geräten im Verhältnis zu Pics und Triedern.

Neben einigen größeren Exemplaren wurde auch ein kleines, flaches Quarzitgeröll (ca. $70 \times 50 \times 20 \mathrm{~mm}$ ) gefunden, das auf beiden Seiten an den Kanten grob beschlagen ist. Es erinnert an die Fundstücke von Cannstadt (WAGNER 1986: 204, 205).

Bei den Choppern und Chopping Tools handelt es sich um einfache, mit wenigen Schlägen bearbeitete Stücke. Bemerkenswert ist ein größeres Chopping Tool aus Kevelaer; seine Maße sind $139 \times 117 \times 75$ mm, es weist 8 Negative auf (vgl. Taf. 6, Fig. 2).

\subsubsection{Polyeder}

Es wurden bisher 3 Exemplare gefunden, die nur teilweise bearbeitet und stark verrollt sind. Bei der Ansprache dieser Stücke ist Kenntnis typischer Acheuléen-Polyeder aus Quarzit hilfreich.

3.5.6 Faustkeile s. 1.; Bifaces, Pics, Trieder, Cleaver (TAvoso 1978: 23, 25)

Faustkeiles. s. = Bifaces:

Aus Kirchhellen liegen 3 Bifaces vor. Das erste Stück, aus Taunusquarzit, Kihe 1, ist dick, im Querschnitt fast quadratisch, alternierend geschlagen; an der Oberfläche sind Cortexreste vorhanden. Die Kanten sind so geschlagen, daß sie das Gerät formen, zum Schneiden jedoch ungeeignet sind, die Spitze ist das Ziel der Herstellung. Das Stück ist abgerollt. Die Maße: $\mathrm{L}=145, \mathrm{H}=60, \mathrm{~B}=72 \mathrm{~mm}$ (Taf. 3, Fig. 2). Biface Kihe 158 ist lang, flach, im Längsschnitt keilförmig, die Spitze ist unsymmetrisch. Das Stück ist aus quarzitischem Sandstein (Karbon?). Eine Seite ist steil, durch wenige kräftige Schläge (wie auch Trieder Kihe 144), die andere Seite ist flach, die Kante durch alternierende Schläge konvex geformt. Die Maße: $150 \times 49 \times 79 \mathrm{~mm}$.

Der Biface 169, aus dem zähen Tertiärquarzit, verrollt und patiniert, hat angenähert mandelförmigen Umriß. Die Spitze ist durch einen quer angesetzten Schlag entweder beschädigt oder zur Meißelschneide umgeformt (wie auch bei Trieder Kihe 144 und Triederspitze 183). Die Unterseite ist dachförmig zurechtgeschlagen, ein Teil davon Cortex. Die Oberseite ist von einer Rippe schief durchzogen. Das Stück erinnert an die unsymmetrischen Stücke des Bergerac, etwa Typ Cantalouette, Le Pendu (GUICHARD 1976: 921-923). Wir begegnen dieser auffälligen Asymmetrie bei den Stücken aus zähem Tertiärquarzit immer wieder. Eigene Schlagversuche fördern das Verständnis.

Tried e r :

Aus beiden Betrieben liegen jeweils mehrere Trieder sowie Bruchstücke vor. Die kompletten Stücke sind über die ganze Länge dreieckig geschlagen, manchmal nur zweiseitig. An anderen Exemplaren ist etwa die 
Hälfte des Körpers beschlagen. Verwendet wurden alle vorhandenen Gesteine. Die Spitze ist gelegentlich alt oder neu abgebrochen (vgl. Taf. 1, Fig. 1 und Taf. 2, Fig. 1a).

Ein Stück aus rötlichem quarzitischem Sandstein ist bemerkenswert. Die Spitze wurde durch zwei kräftige Schläge geformt, ist hochrückig-lang, schmal, dreieckig-schnabelförmig, der vorderste Teil alt abgebrochen. Der Talon ist ebenfalls beschlagen. Dieses Stück leitet über zu einem sehr dicken Terra-AmataPic mit abrupter Retusche (vgl. BIBERSON 1961: 141 sowie AGUADO 1963: Taf. XIV und XV).

Unspezifische Pics:

Hier wurden Stücke zusammengefaßt, die sich keiner Gruppe zuordnen lassen. Dazu gehören Stücke aus zähem Tertiärquarzit: der Körper ist völlig unregelmäßig, rundlich-barrenförmig und läuft in einer kurzen, dicken, leicht gebogenen Spitze aus; diese ist zersplittert. Aus Taunusquarzit sind Stücke, an denen durch einige Schläge eine kurze, dornartige Spitze herausgearbeitet wurde. Geräte dieser Art sind z. B. im Inventar von Pinedo häufig (Sammlung im Nationalen Archäol. Museum Madrid, eigene Beobachtung).

$\mathrm{Pic}$ a $\mathrm{lt}$ è $\mathrm{rne}$ :

Ein Exemplar, geschlagen aus einem flachen Geröll, wurde in Kevelaer gefunden. Die Spitze ist eine schräge Schneide.

P i c sur dièd re cor t ic a l:

Diese Gruppe ist durch eine Reihe von Fundstücken vertreten. Gelegentlich ist auch eine der beiden (normalerweise Natur-) Dieder-Flächen teilbearbeitet, meist sind es jedoch cortexbedeckte Flächen an einem kantigen oder plattigen Geröll. Typisch für diese Geräte ist, daß sie fast immer einige unregelmäßig verteilte Schlagnegative am Körper aufweisen. (Neben der bearbeiteten, gewölbten, den Pic bildenden Oberfläche.) Beispiele Sammlung Pinedo, M.A.N. Madrid (Taf. 1, Fig. 2). Aus der Reihe der normal großen Stücke von ca. $120-140 \mathrm{~mm}$ Länge ragt ein Exemplar heraus, das an einem plattigen Taunusquarzit-Geröll mit natürlicher, dachartiger Kante geschlagen wurde: das Stück wiegt $2,1 \mathrm{~kg}$, seine Maße sind $\mathrm{L}=251, \mathrm{H}=126, \mathrm{~B}=95 \mathrm{~mm}$ - eine Größe, die in Mittel- und Alt-Acheuléen-Inventaren nicht auffällt.

Terra Amata-Pic mit abrupter Retus c he :

Bei unseren Fundstücken handelt es sich um eine Gruppe von einfachen, meist asymmetrisch geformten Geräten mit einer dreieckigen Spitze, während der Support (Geröll, Trümmerstück, Platte) unter- schiedlich ausgebildet ist, daher eine auffällige Variationsbreite in der Form (vgl. Tafeln in SANTONJA \& PEREZ-GONZALES 1984, 147, 148). Diese Geräte sind teilweise schwierig zuzuordnen, es gibt Übergänge so zum "chopper à point" (vgl. Taf. 3, Fig. 1 und Taf. 5, Fig. 2).

\section{Cle aver:}

Diese Geräte sind auf unseren Fundstellen selten. Auf jeder wurde je ein Cleaver-artiges Gerät gefunden, beide am Abschlag, beide aus dem zähen Tertiärquarzit. An einem der Geräte wird die Arbeitskante durch das Zusammentreffen eines Negativs mit der Cortexfläche des Gerölles, am anderen durch das Treffen von zwei Negativen gebildet. Die Umrisse sind nur spärlich modifiziert, sie entsprechen eher den "cleaverflakes" von ISAAC (1967: 247) (vgl. Taf. 2, Fig. 2).

Inventar der beiden Fundstellen: (Stand 4. 2.92.)

$$
\text { Liste der Inventare }
$$

$\begin{array}{lcc} & \text { Weeze } & \text { Kirchhellen } \\ \text { Bifaces } & 4 & 2 \\ \text { Pics } & 16 & 25 \\ \text { Cleaver-artige } & 1 & 1 \\ \text { Chopper } & 7 & 5 \\ \text { Chopping Tools } & - & 4 \\ \text { Kerne } & 16 & 12 \\ & & \text { (incl. 2 Polyeder) } \\ \text { Abschläge } & 10 & 9 \\ \text { Diverse } & 12 & 7 \\ & 66 & 65\end{array}$

Wegen der vermutlich stark selektiven Fundsituation wird auf weitere Auswertung wie Berechnung der Prozentsätze verzichtet.

\subsection{Diskussion der Fundumstände}

Bei dem Bestreben, die problematischeren Umstände an den beiden Fundstellen zu erkennen, sind neben der Abrollung und Oberflächenveränderung der Artefakte eine Reihe weiterer Faktoren in Betracht zu ziehen:

a) Das Vorkommen von überwiegend größeren, schwereren Fundstücken deutet auf eine Sortierung durch den Fluß und auf einen gewissen Transportweg.

b) Artefakte aus Quarz oder Flint dürften meist über die Grenze der Erkennbarkeit hinaus verändert worden sein. Die wenigen Fundstücke zeigen sie an, ihr ursprünglicher Anteil an den Inventaren muß offen bleiben. 
Dies trifft auch auf Sandstein als Rohmaterial zu. Es liegen, neben wenigen deutlichen Artefakten, eine Reihe artefaktverdächtiger Stücke vor, die vorerst gesammelt, jedoch nicht in das Inventar aufgenommen werden. Sandstein (kein „quarzitischer Sandstein”) ist im Altpaläolithikum in größeren Mengen als Rohmaterial verwendet worden, so z.B. in in Marokko BiBERSON (1961: 133) "grès feldspathique".

c) An Taunus- und zähem Tertiärquarzit sind Schlagmerkmale besser als an den übrigen Gesteinen zu erkennen, was Selektion beim Sammeln unterstützt.

d) Einmal erkannte Formen werden beim Sammeln besser wiedererkannt, was ebenfalls Selektion fördert.

e) Von mehreren Autoren wird auf ein nachträgliches Einlagern von jüngeren Objekten in ältere Terrassen hingewiesen, z. B. von KUKLA (1975: 157). Dies setzt aber einen auf der Terrasse fließenden Strom voraus. Die Niederrheinische Bucht begann sich jedoch gegen Ende der Bildung der Jüngeren Hauptterrasse in stärkerem Maße zu heben, sodaß sie in weiten Teilen trocken fiel. Die Artefakte sind abgerollt, müssen also vorher d.h. während der Terrassenbildung geschlagen worden sein. (KIOSTERMANN 1988: 47)

In diesen Fragenkomplex: Einlagerung von jüngeren Objekten in ältere Ablagerungen, gehört auch die Möglichkeit von jüngerer Rinnenbildung. In England wird in Flußterrassen immer wieder die sog. "Channell"-Bildung beobachtet, jüngere Rinnen mit teilweise hoher Artefaktführung (MACRAE \& MOLONEY 1988: 138). An unseren Fundorten konnte jedoch nichts Vergleichbares beobachtet werden. Es gibt viele kleinere Rinnen von 2-10m Breite und $0,1-0,5 \mathrm{~m}$ Tiefe, sie liegen jedoch immer im Terrassenstapel und sind von horizontalen Schichten diskordant überlagert.

In der Kiesgrube Weeze sind lange Profilfronten (etwa $1 \mathrm{~km}$ ) aufgeschlossen, sodaß derart auffällige Bildungen wie die "Channels" in England gar nicht zu übersehen wären; es wird von wesentlich größeren Bildungen berichtet, welche die Terrasse unterbrechen und sich auch deutlich von ihr unterscheiden (MACRAE \& MOLONEY 1988: 168).

f) Der verschiedenartige Grad der Abrollung bzw. Oberflächenveränderung wurde früher häufig als Kriterium für das Vorhandensein verschieden alter Artefaktserien betrachtet. Dies dürfte jedoch nur unter ganz bestimmten Bedingungen zutreffen. In der Auswertung der Grabung Pinedo interpretiert SANTONJA die Abrollung lediglich als Ausdruck unterschiedlicher, von diversen Faktoren beeinflußter Transportwege (QUEROL \& SANTONJA 1979: 159), was hier auch angenommen wird. Die Artefakte können jedoch teilweise nur einen sehr kurzen Weg zurückgelegt haben, was aus dem Vorhandensein guterhaltener Stücke zu schließen ist: kurzer Weg und/oder schnelle, schutzbietende Einbettung. Die stärker abgerollten Stücke können dann auch nicht aus enormen Entfernungen kommen.

g) Im Rahmen der Forschung in Ostafrika wurde festgestellt, daß im Lebensraum des Frühmenschen ein dünner „Schleier" von Artefakten ("scatter”) die Landschaft überzieht (ISAAC 1978: 199). Auf den rohstoffreichen Flußterrassen dürfte, neben Fundkonzentrationen, dieser „Schleier” eher noch stärker sein. In diese Kategorie könnten unsere Funde vielleicht gehören. Eine andere Möglichkeit wäre weitgehende Vermischung des ursprünglichen Bestandes von Konzentrationen und dem „Schleier” durch Hochwasser und Flußtransport mit dem Geröll.

h) In den jüngeren Industrien, wie z. B. dem JungAcheuléen, gibt es deutlich erkennbare Vorformen oder Halbfabrikate (Markkleeberg: BAUMANN \& MANIA 1983: 196-219; Reutersruh: LUTTROP \& BOSINSKI 1971: 134-143). Je älter die Inventare, je schwieriger dürfte diese Unterscheidung werden. In Arbeiten über Alt-Acheuléen-Fundstellen (Ubeidya: STEKELIS et al. 1966; Olduvai: LEAKEY 1971; Tarn/Agout: TAVOSO 1978) werden keine Vorformen beschrieben, im Text wird lediglich vereinzelt darauf Bezug genommen, so LEAKEY (1971: 31 oder 126). Bei den groben Formen, der archaischen Technik sowie unserer Unkenntnis der damit ausgeführten Arbeiten ist heute nicht mehr zu klären, wieweit unter unseren Stücken Vorformen sind. Bei zwei Artefakten, deren Spitzen alt abgebrochen sind (Triederspitze Kihe 183 und Trieder Kihe 172), läßt sich nicht feststellen, ob sie bei der Herstellung oder beim Gebrauch abbrachen.

i) Bei Betrachtung der Fundserie muß man sich die Frage stellen, ob es sich nicht um eine Aufsammlung von durch Naturkräften in täuschender Form beschädigter Gerölle handelt, die einen Fundkomplex vortäuschen; die marginale Bearbeitung der meisten Stücke könnte ein Indiz dafür sein. Wesentliche Argumente sprechen jedoch für die anthropogene Herkunft der Fundstücke.

- Gerade altpaläolithische Fundkomplexe wie z. B. La Bastide de l'Anjou (TAvoso 1978: 327) oder die Günz-Serie von Nancy (JANOT 1981: 307) sind derart marginal bearbeitet. Dies dürfte mit der jeweiligen Situation (kurzfristige Begehung?) und dem verfügbaren Rohstoff (hier plattig oder unregelmäßig geformten Geröllen aus schwer zu schlagendem Material) zusammenhängen.

- Es liegen eine Reihe deutlicher, mit 6-9 Negativen versehener Fundstücke vor. Den marginal bearbeiteten Stücken könnte, falls sie als Einzelfund vorlägen, kein Artefaktcharakter zugesprochen 
werden. Im Komplex stützen sich die Stücke jedoch. Sichere Fundstücke sind:

Trieder Kihe 140
Pic Kihe 190
Chopper Kihe 190
Terra Amata Pic KgWe 185
Chopping Tool KgWe 185
Chopper KgWe $148,75,2$ und 1
Kerne aus Weeze: KgWe 10,13 und 158

Ein Sammler, der seine Fundstelle über längere Zeit beobachtet, lernt den Geröllbestand und die Formen gründlich kennen. Er kann daher weit besser als ein Außenstehender beurteilen, welche Formen und Beschädigungen natürlich sind und häufig vorkommen oder ungewöhnlich sind. Dies hilft bei der Beurteilung der Fundstücke. Als Vergleichsmaterial wurden zwei Kollektionen angelegt, die zur Abgrenzung dienen:

- Geofakte, natürliche Trugformen, Stücke aus der "Grauzone"

- Natürlich entstandene Dreiecksformen und Spitzen

Daneben wurden Abschläge sowie bearbeitete Stücke aus eigener Schlagarbeit, aus verschiedenem Material, aufbewahrt.

Eine verbreitete Ansicht fordert, Fundstücke mit modifizierter Oberfläche nur dann zu akzeptieren, wenn andere Belege für einen urgeschichtlichen $\mathrm{Zu}$ sammenhang wie Knochenansammlung, Feuerspuren o. ä. zusätzlich vorhanden sind: ohne solche sei das Entstehen von Artefakten eher unwahrscheinlich. Dem steht inzwischen eine Serie reicher Flußterrassenfundstellen im Mittelmeerraum gegenüber, die ohne andere urgeschichtliche Belege tausende von Artefakten, teils mehr, teils weniger verrollt, geliefert haben. (Querol \& Santonja 1979; Biberson 1961; Tavoso 1978).

Bisher wurden 14 Kiesgruben begangen, zwei weitere sind aus der Beschreibung der bearbeitenden Sammler bekannt. Lediglich 5 führen Artefakte, eine davon ein Jung-Acheuléen. Wären die Fundstücke von Weeze und Kirchhellen Naturprodukte, sollten sie überall vorkommen.

j) Gewisse Gerölle mit Spuren möglicher Bearbeitung wurden grundsätzlich wegen des hohen Risikos natürlicher Beschädigung nicht gesammelt: alle dünnen, flachen Stücke, deren Kanten bearbeitet erschienen (dünne, flache Stücke können zu leicht an den Kanten brechen) sowie alle Stücke mit scheinbarer Retusche, da diese bei bewegtem Geröll zu leicht entstehen kann: eine Abgrenzung gegen menschliche Bearbeitung ist nicht sicher möglich. k) Bei der Untersuchung der Fundstücke wurden folgende Kriterien angewandt:

- die Form sollte eine Funktion anzeigen und aus eigener Anschauung und/oder Zeichnungen bekannt sein. Die Kenntnis einer Reihe entsprechender Sammlungen grenzt die Gefahr ein, echte Artefakte fälschlich auszuscheiden.

- es sollten wenigstens $2-3$ Negative erkennbar sein, jedoch entscheidet der Gesamteindruck.

- bei den Abschlägen wurde Wert auf erkennbaren Schlagpunkt und Zertrümmerungszone gelegt. Der Schlagflächenrest ist eher groß bis sehr groß. Es ist zu berücksichtigen, daß bei gröberen Quarziten Schlagmerkmale häufig nur schwach ausgeprägt sind; Erfahrungen mit diesem Material und der Gesamteindruck muß entscheiden helfen.

\subsection{Die altpaläolithischen Funde vom Niederrhein im Vergleich zu anderen west- und mitteleuropäischen Inventaren}

Die Inventare von Kevelaer und Kirchhellen zeigen in den Variationen faustkeilartiger Geräte, besonders der Trieder, Ähnlichkeit mit dem Abbevillien. In der archaischen Schlagtechnik sind Zusammenhänge mit den Funden von Winningen/Bisholder (BERG \& FIED. LER 1983), aber auch den älteren Inventaren von Lothringen erkennbar (JANOT 1981, 1988). Zu dem älteren Fundserien des französischen Mittelmeerraumes (Labastide d'Anjou, Le Rivalou, Terrassen des Tet: TAvoSO 1978 und Collina-Girard 1975) bestehen Parallelen im Vorkommen von Pics sur dièdre cortical oder Bifaces partiell sowie generell der äußerst sparsamen und groben Bearbeitung der Stücke (Mitteilung Fiedler).

Besonders auffällig ist die Ähnlichkeit von Varianten der Terra-Amata-Pic-artigen Geräte mit den namengebenden Stücken des Fundplatzes in Nizza, aber auch, neben den Pics sur dièdre cortical, mit den entsprechenden Fundstücken von Pinedo; dasselbe trifft auch auf die Trieder und Pics von Sidi Abderrahman/ Marokko als Fundstelle des Mittelmeerraumes zu. (Eigene Beobachtung an den entsprechenden Museen in Nizza, Madrid, Paris)

Das Fundmaterial von Pinedo umfaßt die verschiedenen Erhaltungszustände in variantenreicher Form; es bildet aber auch ein heterogenes Feld, in dem die Abgrenzung der verschiedenen Typen oft schwerfällt. Obwohl klassische Faustkeilformen vorkommen, zeigen die vielen Stücke mit grob behauenen, oft stumpfwinkligen Kanten, daß die Hersteller weniger messerähnliche Schneidwerkzeuge als spitze Hackgeräte anfertigen wollten (FIEDLER 1988, 57). Diese Feststellung trifft auch auf andere Alt-Acheuléen-Inven- 
tare zu. Scharfkantige Faustkeile sind nur dort häufiger, wo als Ausgangsstück ein Abschlag und nicht ein Geröll oder Trümmerstück genommen wurde.

Bei Beurteilung der Funde und Vergleich mit anderen Inventaren muß man sich im Übrigen vor Augen halten, daß nicht die Form, wohl aber die Schlagtechnik standardisiert war. Ähnlichkeiten sind daher durch die einfache Technik bedingt.

Die forschungsgeschichtlich späte Entdeckung vorliegender Inventare dürfte mit den Möglichkeiten der Erkennbarkeit derartiger Steingeräte zu tun haben. Klassische Faustkeilformen, wie sie aus dem Jüngeren Acheuléen bekannt sind, fehlen hier völlig. Dafür gibt es Pics, Trieder und Chopperformen. Sie sind in Deutschland erst durch die Arbeiten KRÜGERs (1980) in Münzenberg bekannt geworden. Deren grobe Machart und gewisse technologische Nähe zu möglichen Naturprodukten verlangen nach erfahrener Beurteilung durch Fachleute. Die meisten Einzelstücke sind dabei nicht sicher zu bewerten; erst Fundserien machen eine Beurteilung möglich. Dazu kommt, daß Terrassenfunde erwartungsgemäß starke Veränderungen durch Schottertransport und Abrollung erlitten haben. Abschlagnegative und andere Merkmale der anthropogenen Zurichtung sind daher häufig verschliffen (nicht immer!) und müssen durch den Schleier dieser Veränderungen betrachtet werden. Diese Betrachtungsweise verlangt Kenntnisse, die den meisten Sammlern von Sahara-Faustkeilen oder jedem wissenschaftlichen Bearbeiter von Terrassenfunden in Spanien und Südfrankreich vertraut sind. Bei vielen Archäologen wird es hierzulande verständliche Schwierigkeiten geben, da sie mit solchem Material bisher kaum Berührung hatten.

Mit dem notwendigen Interesse und etwas Geduld sind dafür jedoch objektivierbare Einsichten zu gewinnen. Funde dieser Art verlangen aber auch immer wieder nach kritischer Abgrenzung, da die Spanne zwischen erkennbaren technologischen und typologischen Merkmalen sowie verwaschenen möglichen Kennzeichen breit ist, sodaß die Gefahr besteht, nichtartifizielle Stücke mit in die Inventare aufzunehmen.

\section{Bewertung}

Die Befunde von Kevelaer und Kirchhellen sind eine faustkeilreiche Variante des im westlichen Europa bekannten frühen Acheuléen. Durch die glücklicherweise klaren geologischen Fundbedingungen sind diese Inventare, wenn auch in sekundärer Lagerung, bemerkenswerte Zeugnisse der Kultur frühester Menschen in Europa. Gegenüber den größeren Möglichkeiten einer Fundstelle in primärer Lagerung ist ihre
Aussage begrenzt. Neben dem Nachweis über die Anwesenheit des Frühmenschen besteht aber durch langfristige Beobachtung die Möglichkeit, Fundserien zu bilden, die typologische Hinweise geben können; hierin liegt jetzt, im 2. Schritt, der eigentliche Wert der Fundstellen.

\section{Anmerkungen}

Meine Arbeit wurde durch eine Reihe von Institutionen und Personen unterstützt: durch Genehmigungen, direkte Hilfe, Beratung, konstruktive Kritik und förderndes Interesse, für die ich danke. Frau G. BARNICK schrieb das Manuskript; In verschiedener Form halfen mir: Prof. G. BOSINSKI, Neuwied; Frau CACHO vom Museo Arqueologico Nacional in Madrid; Frau GoUDET vom Terra-AmataMuseum in Nizza; A. HeINRICH vom Museum für Ur- und Ortsgeschichte Bottrop; F. JANSEN, Geolog. Landesamt NW, Krefeld; Frau B. KaletsCH, Marburg: Zeichnungen der Artefakte; Dr. J. Klostermann, Geolog. Landesamt NW, Krefeld; G. Rousseau, Musée de l'Homme, Paris; M. SANtONJA GOMEZ, Museo Provincial Salamanca; R. Schmitz, Essen; Dr. E. Schumacher, Essen; Prof. K. Thome, Krefeld.

Ebenso möchte ich den Leitungen der Kiesgruben danken, die mich freundlicherweise in den Betrieben sammeln ließen.

Mein besonderer Dank gilt Herrn Dr. L. Fiedler, Marburg. Ohne seine tatkräftige Unterstützung wäre diese Arbeit nicht geschrieben worden.

\section{Schriftenverzeichnis}

Aguado, M. M. (1963): El Yacimiento prehistorico de Pinedo (Toledo) y su Industria triédrica. Publ. del Inst. Prov. de Invest. estudios Toledanos, serie II, num 1; Toledo.

BaUmanN, W. \& MANiA, D. [Hrsg.] (1983) mit Beiträgen von Toepfer, V. und EissmanN, L.: Die Paläolithischen Neufunde von Markkleeberg bei Leipzig. Veröffentlichungen des Landesmuseums für Vorgeschichte, Dresden, Bd. 16. 280 S., 192 Abb., 33 Taf.; Dresden.

BERG, VAN, A. \& FIEDler, L. (1983): Altpaläolithische Funde von Winningen und Koblenz-Bisholder an der unteren Mosel. Arch. Korrblatt 13: 219-298; Mainz.

Biberson, P. (1961): Le Paléolithique inférieur du Maroc Atlantique. Service des Antiquités du Maroc. 544, 194 pl., 52 fig.; Rabat.

Bordes, F. (1984): Leçons sur le Paleolithique. II und III. Presse du CNRS.II $=459,233 \mathrm{Abb}$; $\mathrm{III}=356,180$ Abb.; Paris.

- (1988): Typologie du Paléolithique ancien et moyen. Presse du CNRS. 112, 108 Taf.; Paris.

BosInSKI, G. (1967): Die mittelpaläolithischen Funde in Mitteleuropa. Fundamente, Reihe A, Bd. 4, 206, 197 Taf., 1 Karte. Köln. 
BRUNNACKER, K. (1978): Gliederung und Stratigraphie der Quartärterrassen am Niederrhein. Kölner Geogr. Arb. 36: 37-58; Köln.

-, FARrokh, F. \& Sidiropoulos, D. (1982): Die altquartären Terrassen östlich der Niederrheinischen Bucht. Z. Geomorph. N. F. 42, 215-226; Berlin.

COLLINA-GIRARD, J. (1975): Les Industries Archaiques sur Galets des Terrasses Quaternaires de la Plaine du Roussillon (P. O., France). These de Doctorat de $3^{e}$ Cycle. Univ. de Provence. 472, 106 fig., 105 pl.; Marseilles.

FIEDLER, L. (1977): Älterpaläolithische Funde aus dem Mittelrheingebiet. Festschrift H. Schwabedissen. Kölner Jahrb. Vor- und Frühgesch. 15. (1975-1977): 13-23; Köln.

- (1985): Zur Formenkunde, Verbreitung und Altersstellung altpaläolithischer Geräte. Quartär 35/36; 81-112; Mainz.

— \& CuBuk, G. (1988): Altpaläolithische Funde von Carmona bei Sevilla und ihre Beziehungen zum Protoacheuléen des Maghreb. In: FiedLeR, L. [Hrsg.]: Kleine Beiträge zur Urgeschichte der Sahara, des Maghreb und der Iberischen Halbinsel. Kleine Schriften aus dem Vorgeschichtlichen Seminar Marburg, Heft 26; 92-139; Marburg.

- (1989): Archaic Artefacts in Hessen, FRG. Early Man News 14; Inst. f. Urgesch. Univ. Tübingen: 33-55; Tübingen.

Guichard, G. (1976): Les Civilisations du Paléolithique inferieur en Perigord: 909-928. In: H. DE LUMLEY [Hrsg.]: La Préhistoire Française. Vol. $1+2 ; 1531$. Paris.

ISAAC, G. L. (1967): The Stratigraphy of the Peninj Group - Early Middle Pleistocene Formations West of Lake Natron, Tanzania: 229-257. In: W. W. BishoP \& J. D. CLARK [Hrsg.]: Background to Evolution in Africa. Univ. of Chicago Press, 935; Chicago.

- (1978): The Olorgesailie-Formation: Stratigraphy, tectonics and the paleogeographic context of the Middle Pleistocene archeological sites: 173-266. In: W. W. Bishop [Hrsg.]: Geological Background to Fossil Man. 585; Scottish Academic Press. Edinburgh.

JANOT, A. (1981): Essai de chronologie des industries paléolithiques à quartzites de la region sud de Nancy (Meurthe-et-Moselle): BSPF 78/10-12: 307-316; Paris.

- (1988): Paléolithique inferieur et moyen de Lorraine, originalité des industries à quartzites. BSPF $85 / 10-12$; 291-303; Paris.

Klostermann, J. (1984) mit Beitrag von J. NÖlting, W. PAAS, H. W. Rehagen: Erläuterungen zu Blatt 4403, Geldern; Geol. Kt. Nordrhein-Westfalen 1:25000. 138, 8 Abb., 13 Tab., 3 Taf.; Krefeld.

- (1988): Quartär; (40-63); In: Geol. Landesamt Nordthein-Westfalen [Hrsg.]: Geologie am Niederrhein. 142, 39 Abb., 4 Tab.; Krefeld.
Kobayashi, H. (1975): The Experimental Study of Bipolar Flakes. 115-128. In: E. Swanson [Hrsg.]: Lithic Technology: Making and Using Stone Tools; The HagueParis.

KRUGGER, K. (1980): Stratigraphisch gesicherte Grabungsfunde zur holsteinzeitlichen Datierung der altpaläolithischen Geröllgeräte-Industrie vom Münzenberger Typ. Fundber. Hessen 17/18: 1-26; Wiesbaden.

KukLA, G. (1975): Loess Stratigraphy of Central Europe. 99-188, In: K. W. ButZer \& G. L. IsAaC [Hrsg.]: After the Australopithecines: 911; The Hague-Paris.

LEAKEY, M. D. (1971): Olduvai Gorge, Vol. 3, Excavations in Bed I und II, 1960-1963; Cambridge Univ. Press. 306, 121 Abb., Cambridge.

LUMLEY, DE H. (1971): Le Paléolithique inferieur et moyen du Midi Mideterranéen dans son cadre Geologique, II: Bas Languedoc-Roussillon-Catalogne. V. supplément à "Gallia Préhistoire". CNRS. 245, 299 Abb.; Paris.

-, De M. A., Miskovsky, J. C. \& Renault-Miskovsky, J. (1976): Le Site de Terra Amata. 15-49. In: H. DE LUMLEY \& L. BarRaL, [Hrsg.] Livret-Guide de l'Excursion B 1. UISPP, IX. Congress.

LuTtrop, A. \& Bosinski, G. (1971): Der Altsteinzeitliche Fundplatz Reutersruh bei Ziegenhain in Hessen. Fundamenta, Reihe A, Bd. 6. 389, 274 Taf., 7 Kart.; Köln.

MAcRaE, R. J. (1988): The Paleolithic of the Upper Thames and its Quartzite Implements: 123-154. In: R. J. MACRAE \& N. MoloneY [Hrsg.]: None-Flint Stone-Tools and the Paleolithic Occupation of Britain. BAR British Series 189: 263, 77 Abb., 41 Taf.; Oxford.

Múller-Karpe, H. (1966): Handbuch der Vorgeschichte, 1. Bd.: Altsteinzeit; 389, 274 Taf.; Beck'scher Verlag. München.

Patterson, L. W. (1983): Criteria for Determining the Attributes of Man-Made Lithics. Journal of Field Arch. 10: 297-307.

Querol, M. A. \& SANTONJA, M. (1979): El Yacimiento Achelense de Pinedo. Excavaciones Arqueologicas en

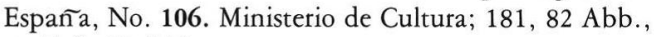
21 Taf.; Madrid.

Rust, A. (1971): Werkzeuge des Frühmenschen in Europa. 71, 29 Abb., Wachholte, Neumünster.

Santonja, M. \& Perez-Gonzalez, A. (1984): Las Industrias Paleoliticas de la Maya I en su Ambito Regional. Excavaciones Arqueologicas en España, No. 135. Ministerio de Cultura, 336, 194 Abb., 3 Taf.; Madrid.

SCHMINCKe, H. V. \& Boogard, v. d., P. (1989): Quartärgeologisch-tephrostratigraphische Neuaufnahme und Interpretation des Pleistozänprofiles Kärlich. Eiszeitalter \& Gegenwart 39: 62-86; Hannover.

Stekelis, H. et al. (1960-66): Archeological Excavationsat Ubeidya. 1960-63: 32, 42 Taf. und 1964-66; 29. 19 Taf. The Israel Academic of Sciences and Humanities; Jerusalem. 
Tavoso, A. (1978): Le Paléolithique Inferieur et Moyen du Haut-Languedoc. Etudes Quaternaires, Mem. No. 5, Univ. de Provence. IHP. 404, 148 Abb.; Paris.

Thévenin, A. (1976): Les Premières industries humaines en Alsace. 810-816. In: H. DE Lumley [Hrsg.]: La Préhistoire Française. Vol. $1+2 ; 1531$. Paris.

Tотн, N. (1987): The First Technology. Scientific American 255 (4): 112-121; New York, N. Y.

WAGNER, K. (1986): Jäger und Sammler im Cannstaedter Travertingebiet. 187-207. In: Landesdenkmalamt
Baden-Württemberg [Hrsg.]: Der Keltenfürst von Hochdorf. Katalog zur Ausstattung in der J. HaubrichKunsthalle Köln, 512 S.; Theiss, Stuttgart.

WÜrgEs, E. (1986): Artefakte aus den ältesten Quartärsedimenten (Schichten A-C) der Tongrube Kärlich, Kreis Mayen-Koblenz/Neuwieder Becken. Arch. Korr. Bl. 16: 1-6; Mainz.

Manuskript eingegangen am 25. 02. 1991. 


\section{Tafel 1}

Kirchhellen: 1. Trieder (Kihe 140) - 2. Pic sur dièdre cortical (Kihe 10 A). - Beide Quarzit.

\section{Tafel 2}

Kirchhellen: 1 a. Trieder von Taf. 1 (Kihe 140). - Weeze: 2. Cleaver (We 165). - Beide Quarzit.

\section{Tafel 3}

Weeze: Terra Amata-Pic (We 102). — Kirchhellen: 2. Biface (Kihe 1). - Beide Quarzit.

\section{Tafel 4}

Weeze: 1. Kern (We 141); Quarz. - Kirchhellen: 2. Abschlag (Kihe 174). - 3. Chopping Tool, Kern (?) (Kihe 175). $-2+3=$ Quarzit.

\section{Tafel 5}

Weeze: 1. Kern (We 10). — Kirchhellen: 2. Terra Amata-Pic (Kihe 5). — Beide Quarzit.

\section{Tafel 6}

Weeze 1a. Kern, von Taf. 5. - Kirchhellen: 2. Chopper (Kihe 213). - Beide Quarzit. 

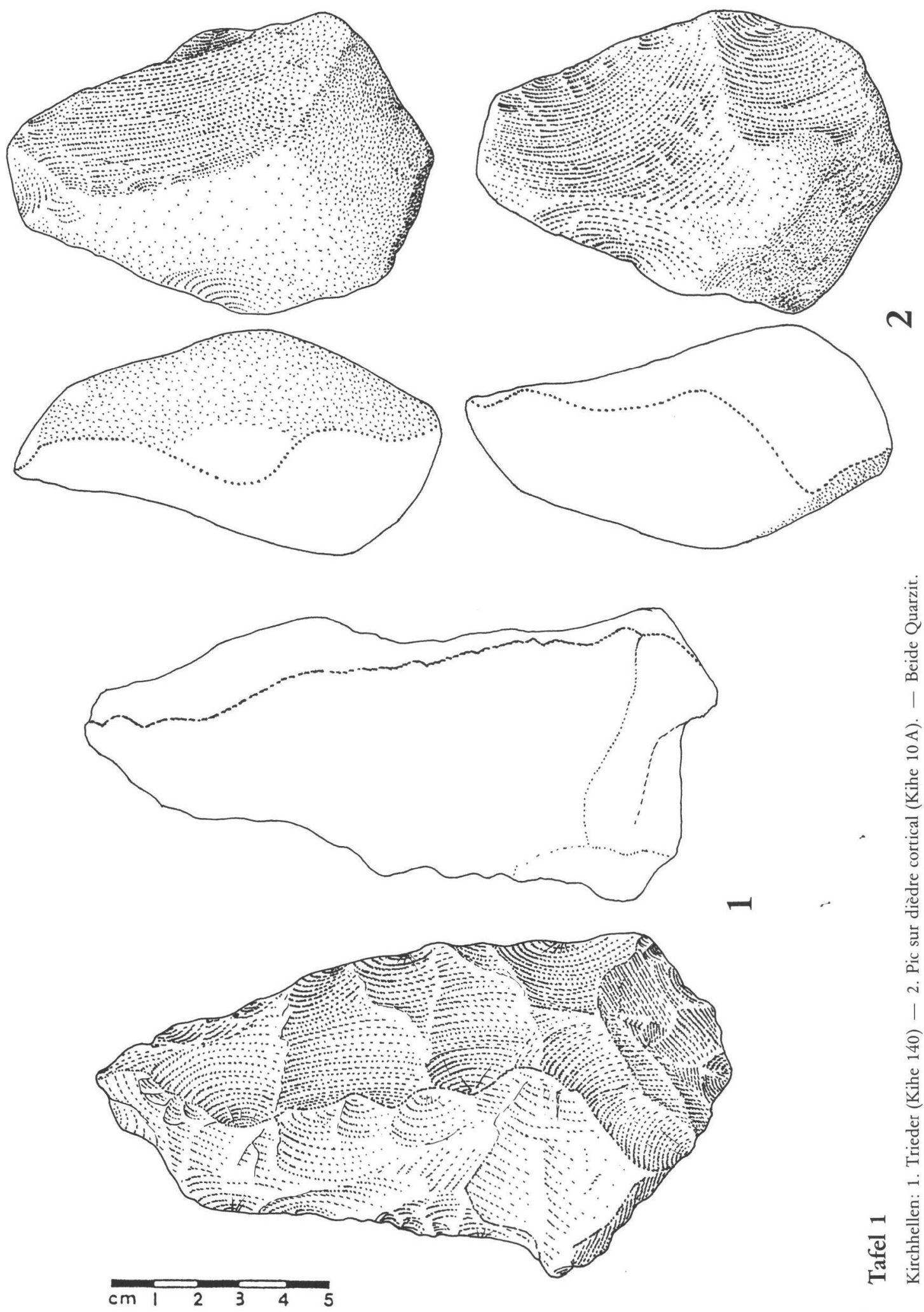


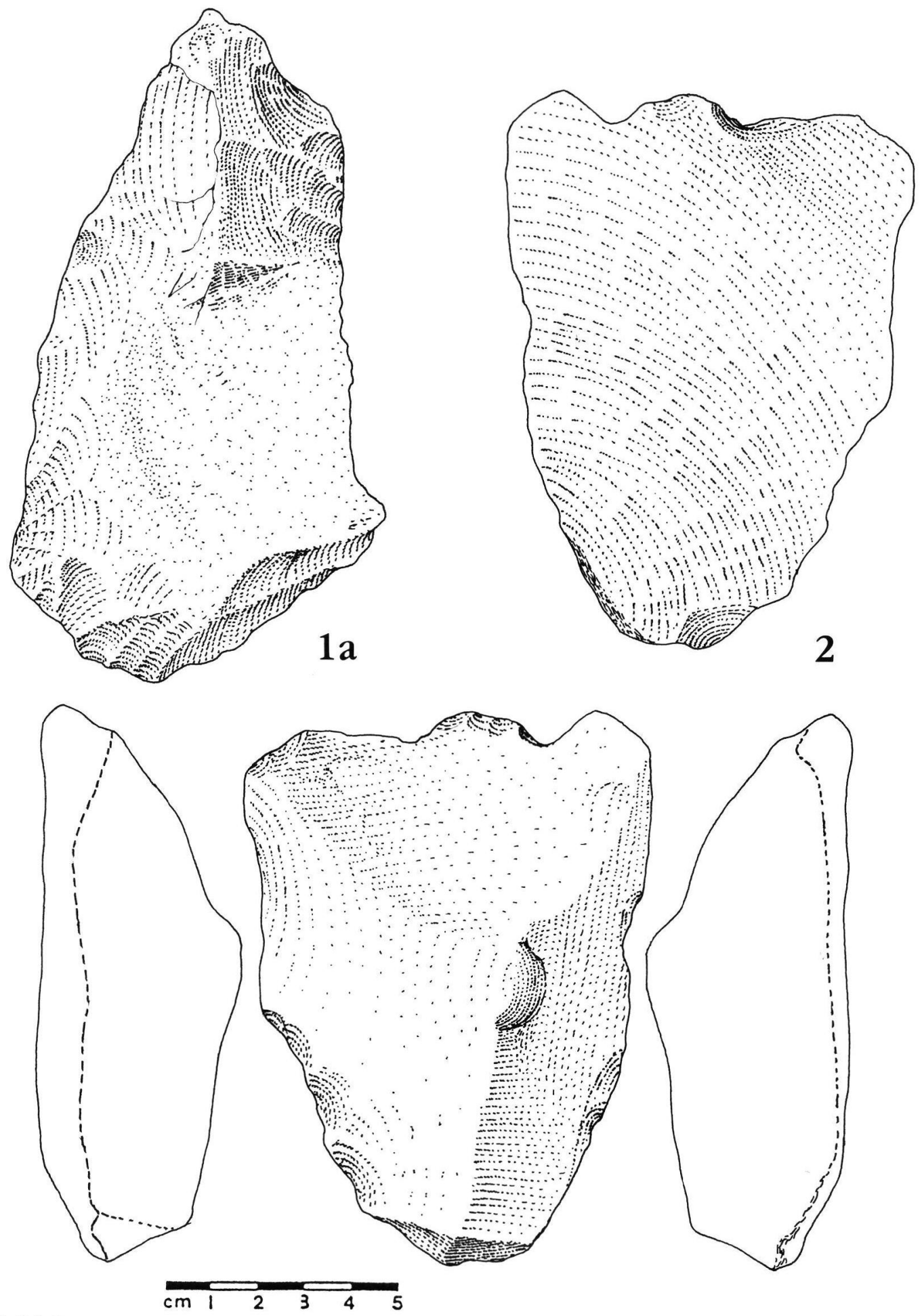

Tafel 2

Kirchhellen: 1 a. Trieder von Taf. 1 (Kihe 140). - Weeze: 2. Cleaver (We 165). - Beide Quarzit. 

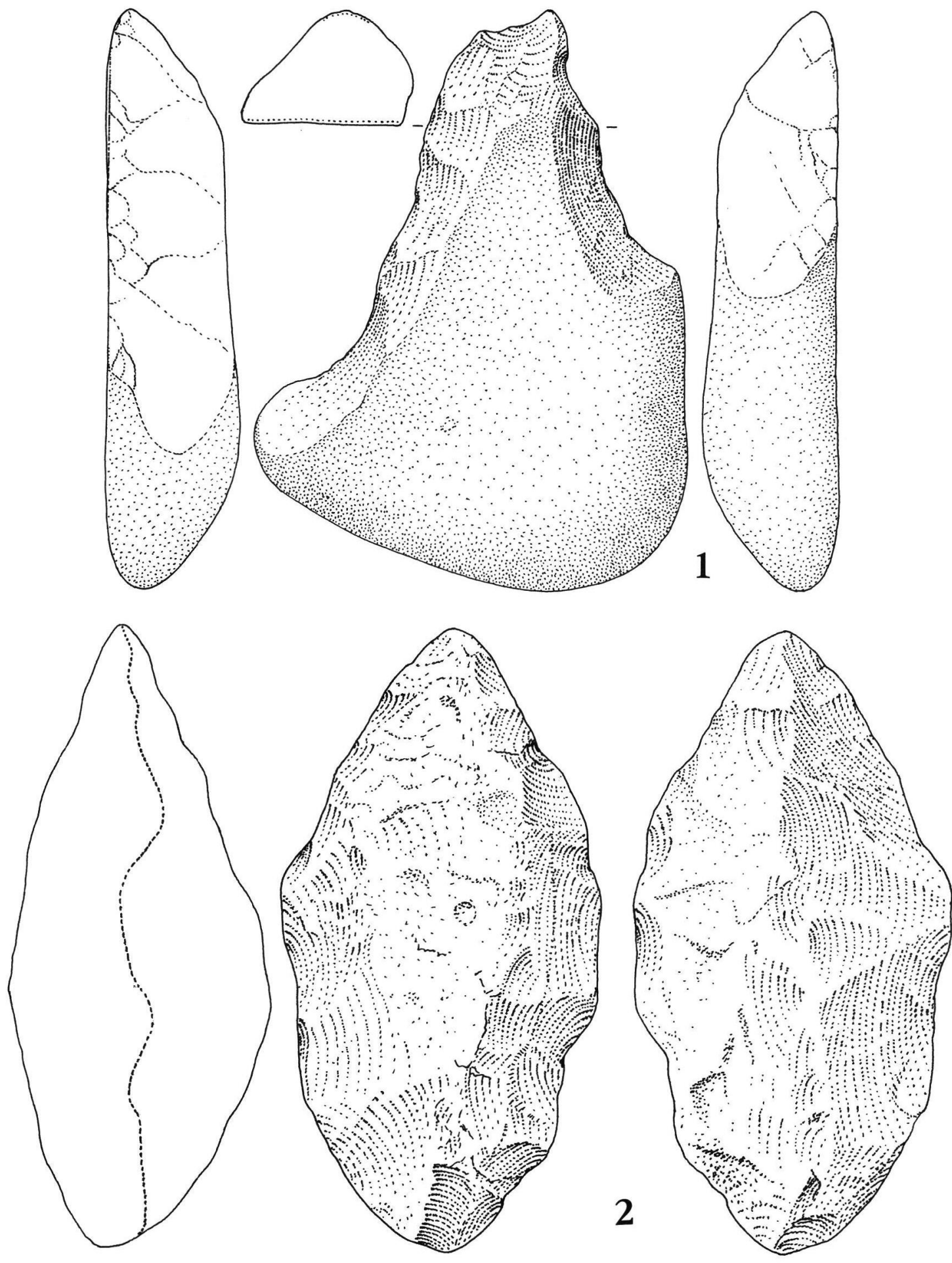

\section{Tafel 3}

Weeze: Terra Amata-Pic (We 102). — Kirchhellen: 2. Biface (Kihe 1). — Beide Quarzit. 

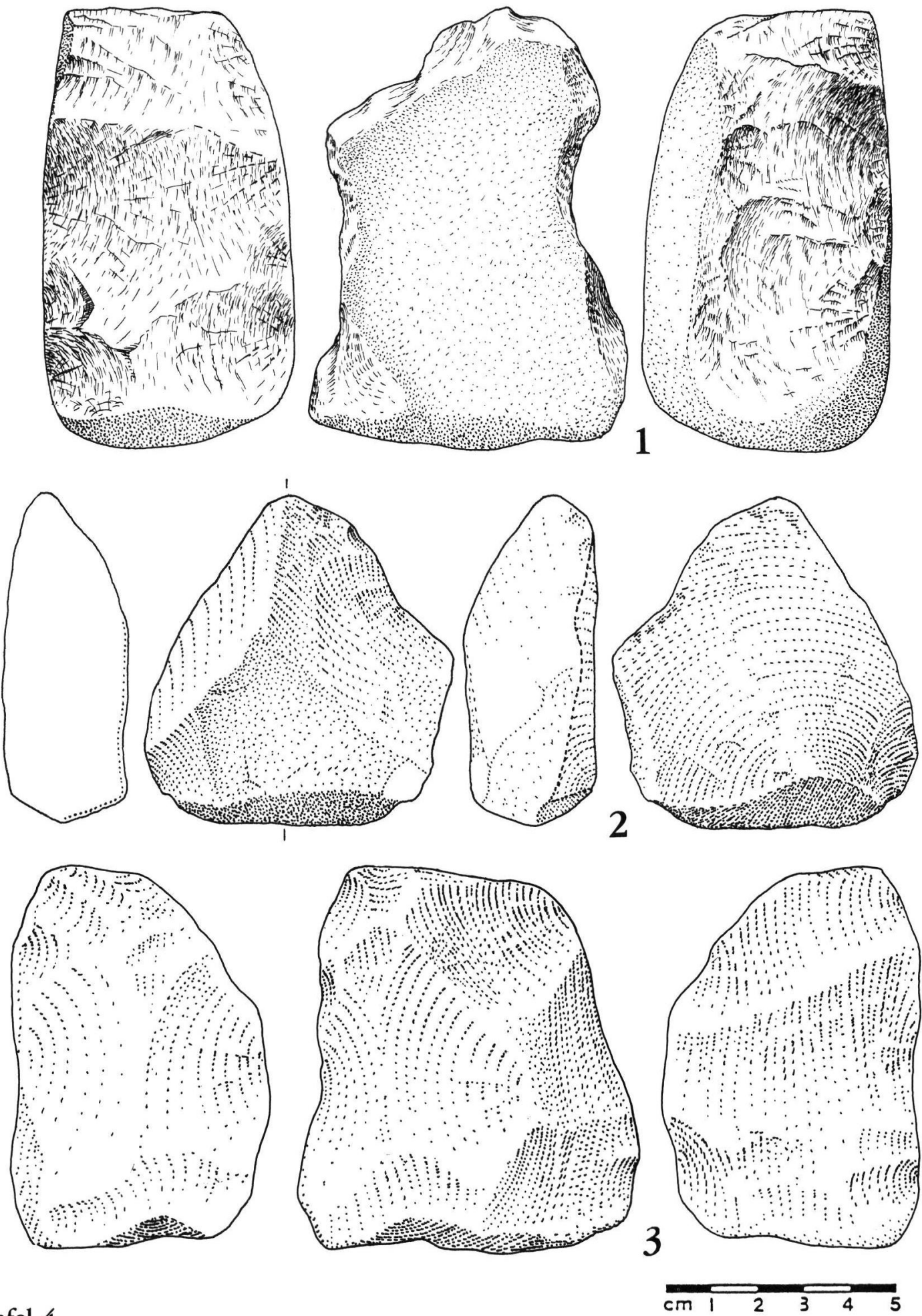

\section{Tafel 4}

Weeze: 1. Kern (We 141); Quarz. - Kirchhellen: 2. Abschlag (Kihe 174). - 3. Chopping Tool, Kern (?) (Kihe 175). $-2+3=$ Quarzit. 

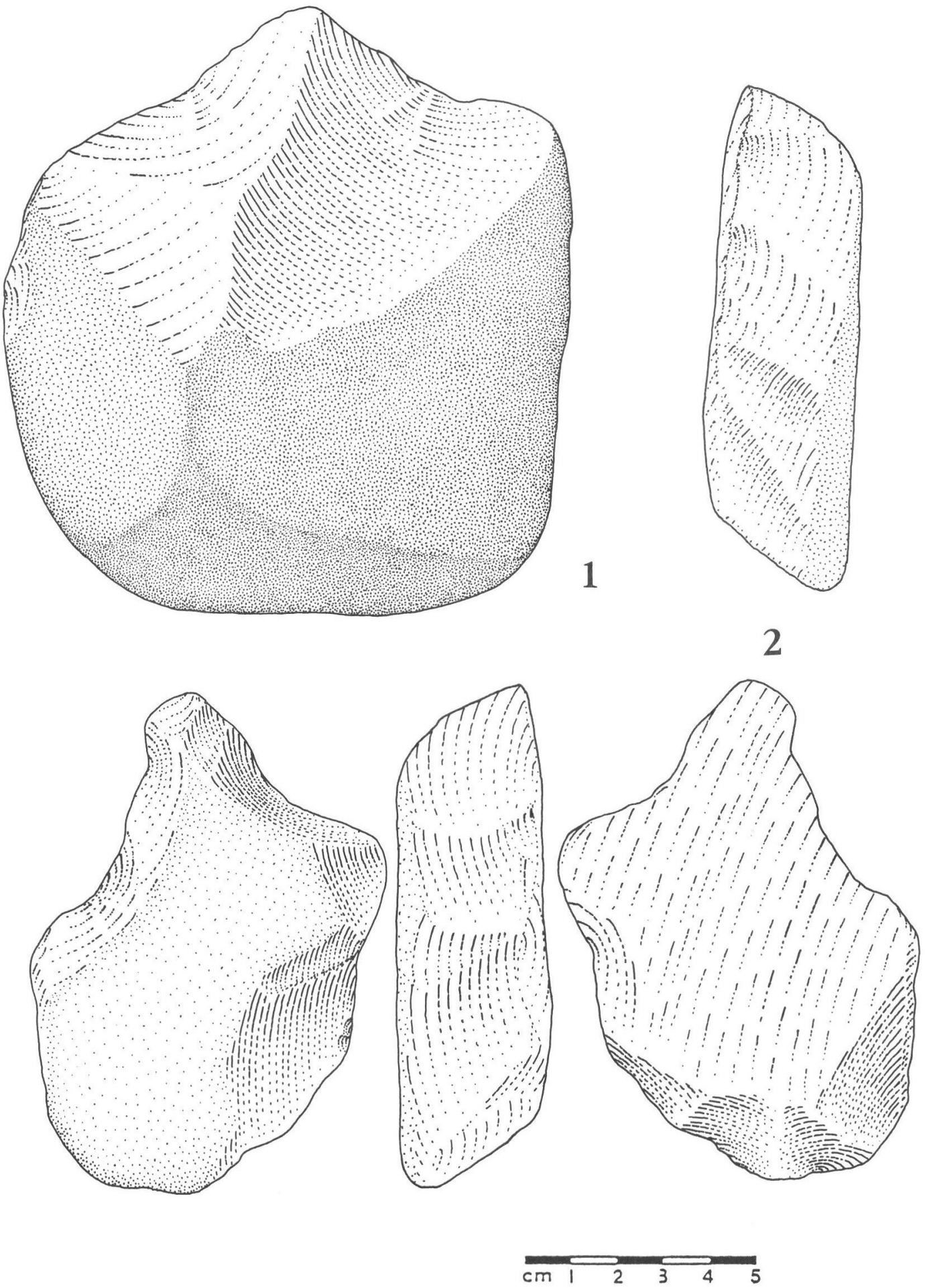

\section{Tafel 5}

Weeze: 1. Kern (We 10). - Kirchhellen: 2. Terra Amata-Pic (Kihe 5). - Beide Quarzit. 

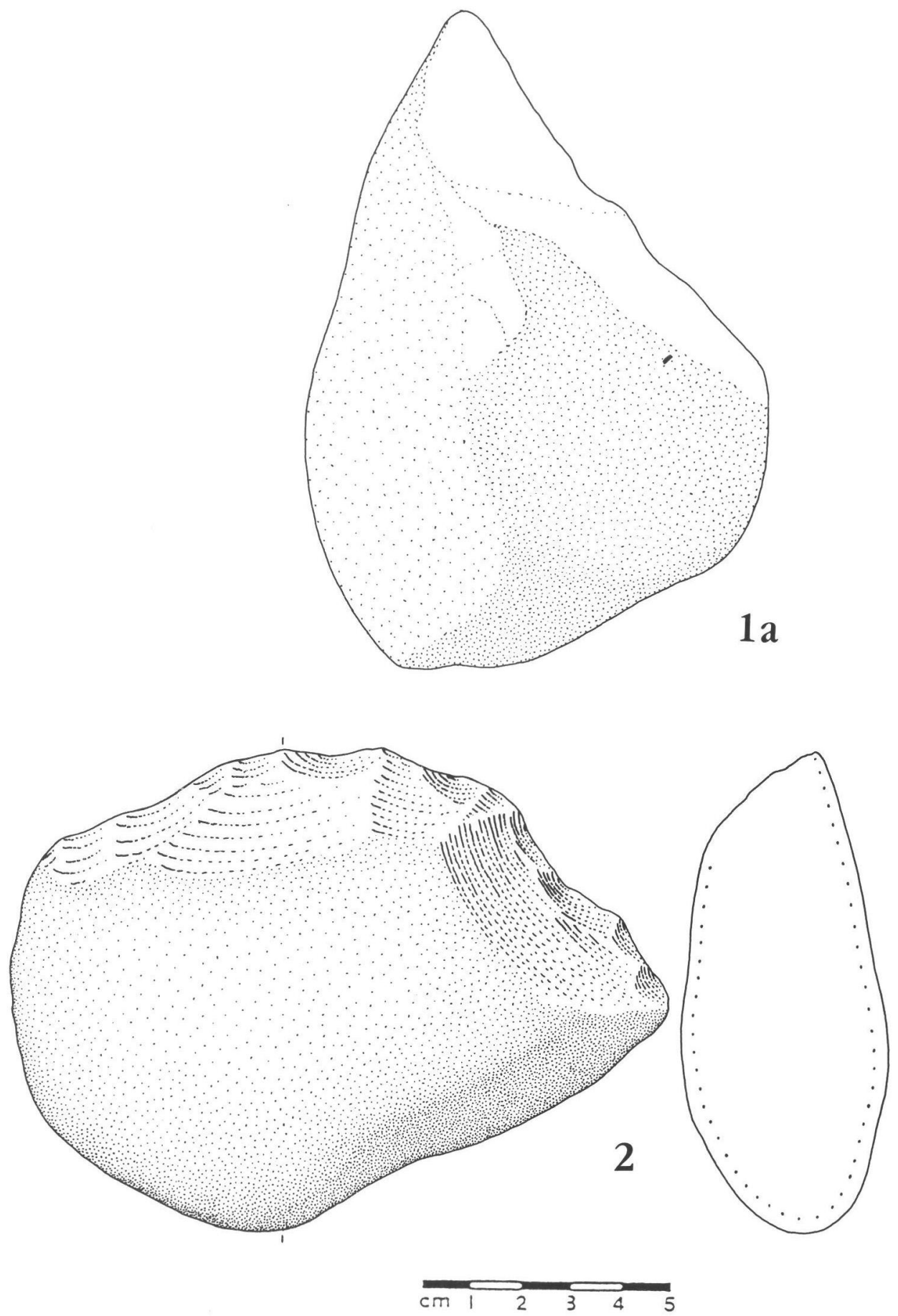

\section{Tafel 6}

Weeze 1a. Kern, von Taf. 5. - Kirchhellen: 2. Chopper (Kihe 213). - Beide Quarzit. 


\section{Tafel 7}

1. und 2: Hauptterrassenkiese und -sande bei Kirchhellen.

\section{Tafel 8}

Kirchhellen: 1) Trieder - 2) Pic: von oben - 3) und 4) Pic: Seitenansicht.

\section{Tafel 9}

Weeze: 1) Chopper - 2) 2 Chopper - 3) Terra-Amata-Pic - 4) Kern. 


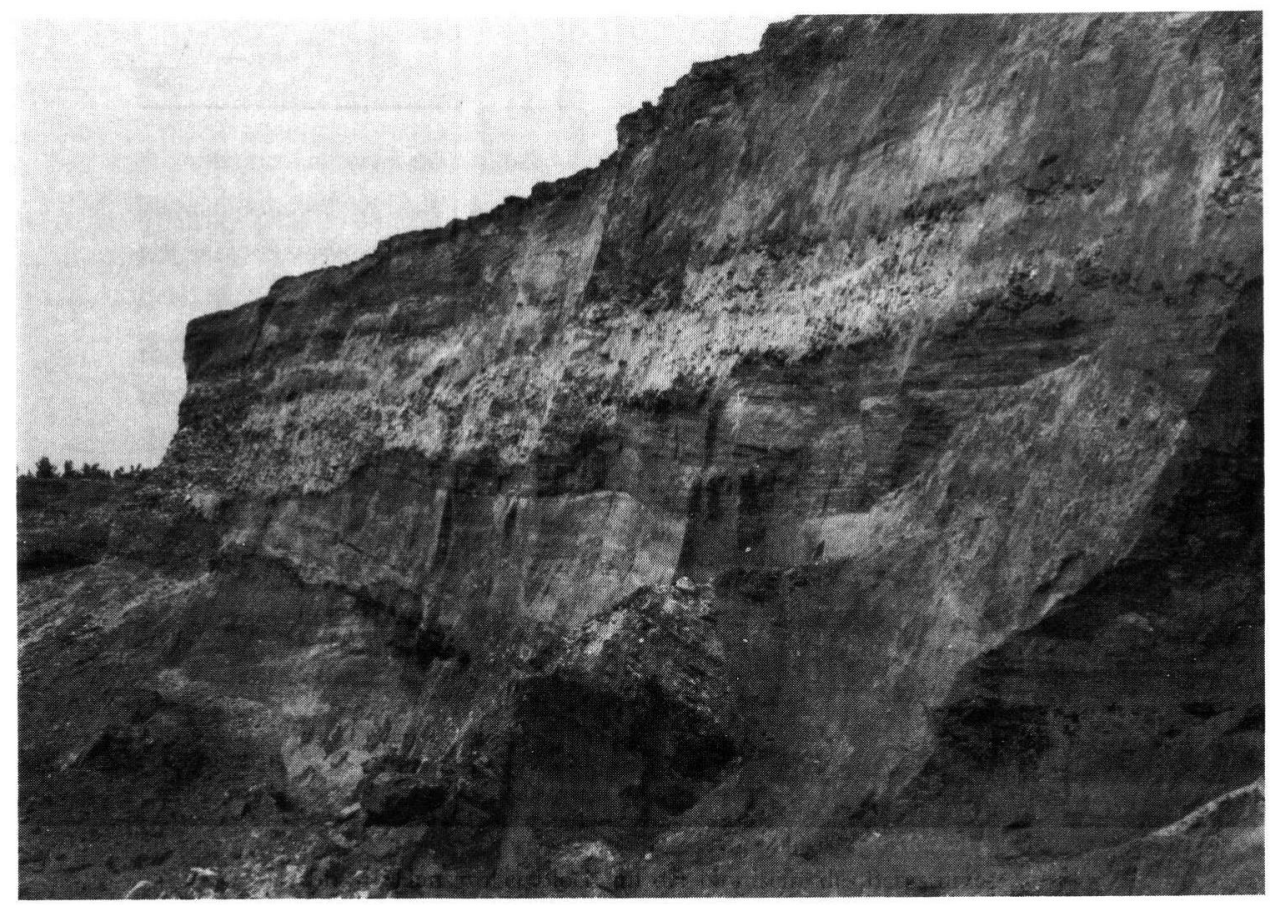

1

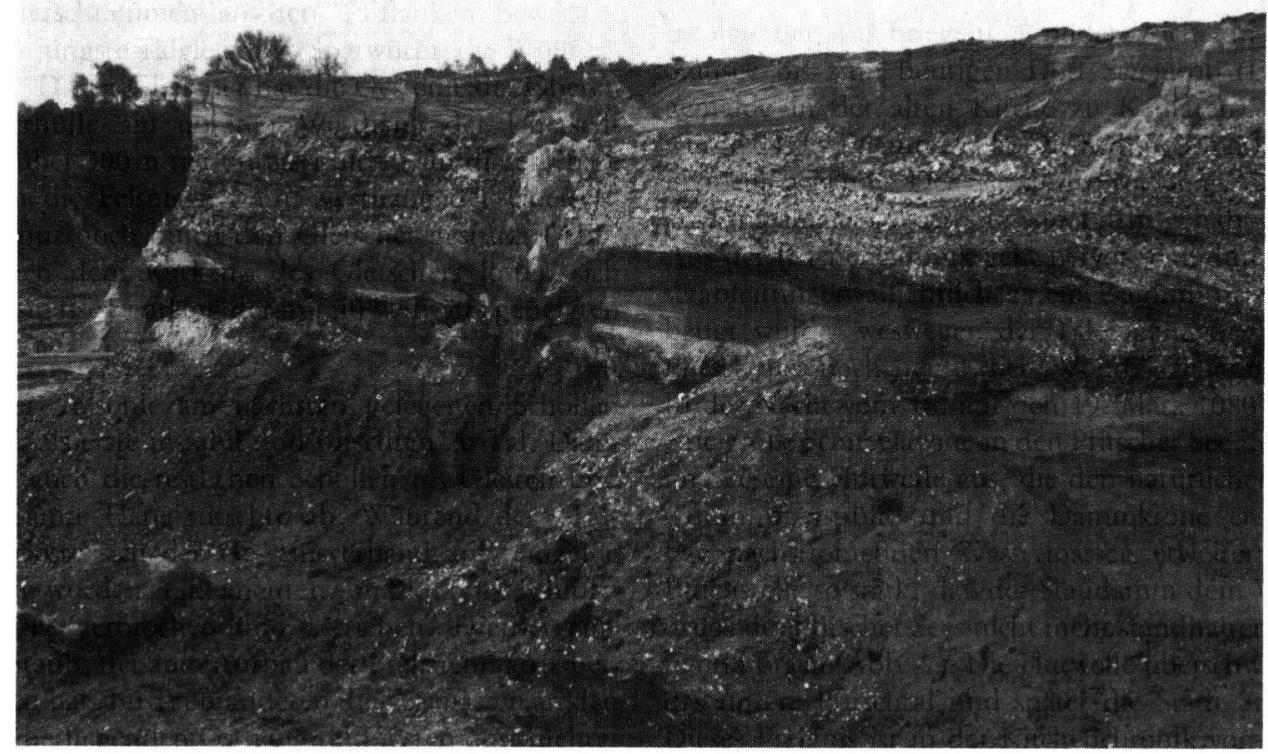



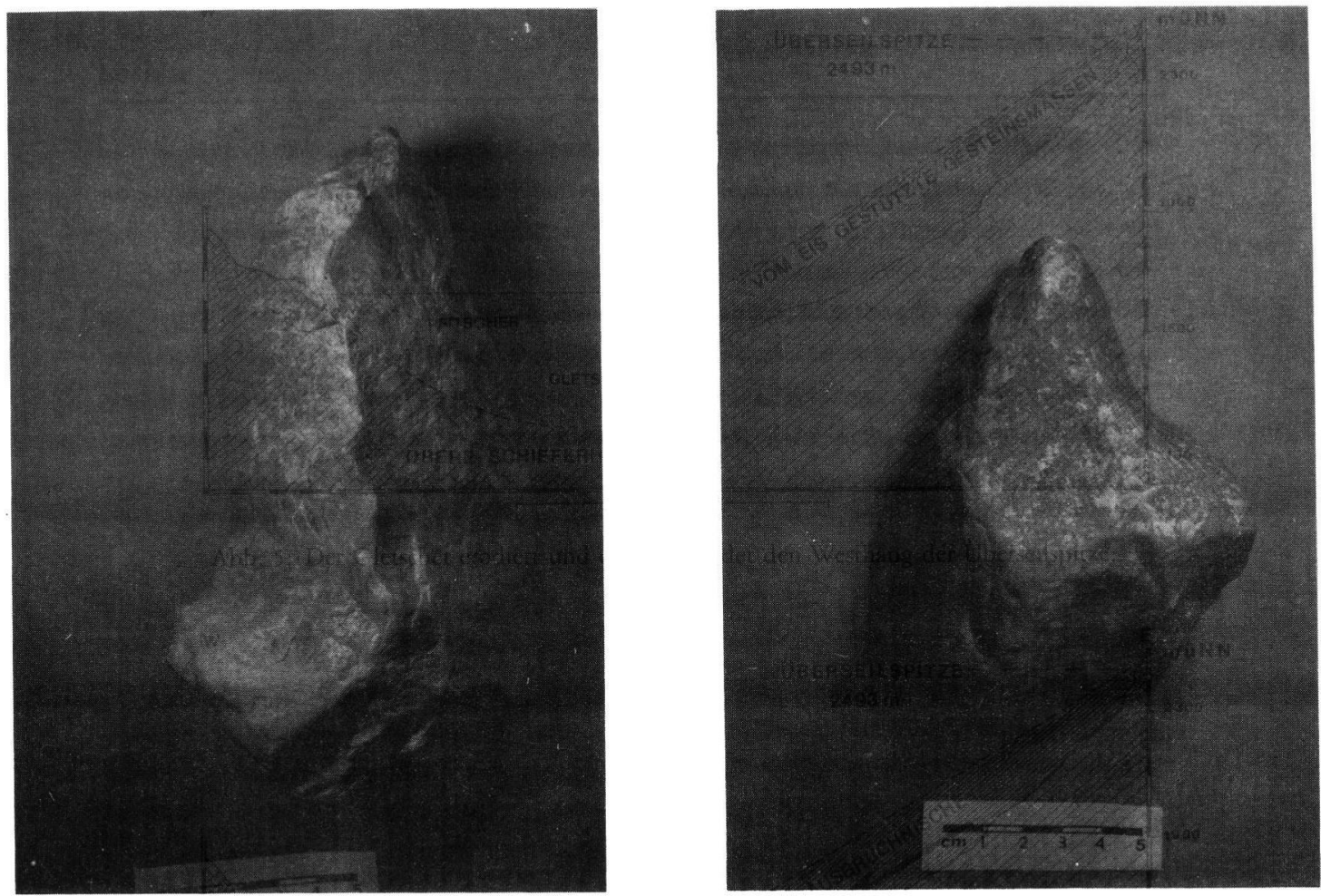

2
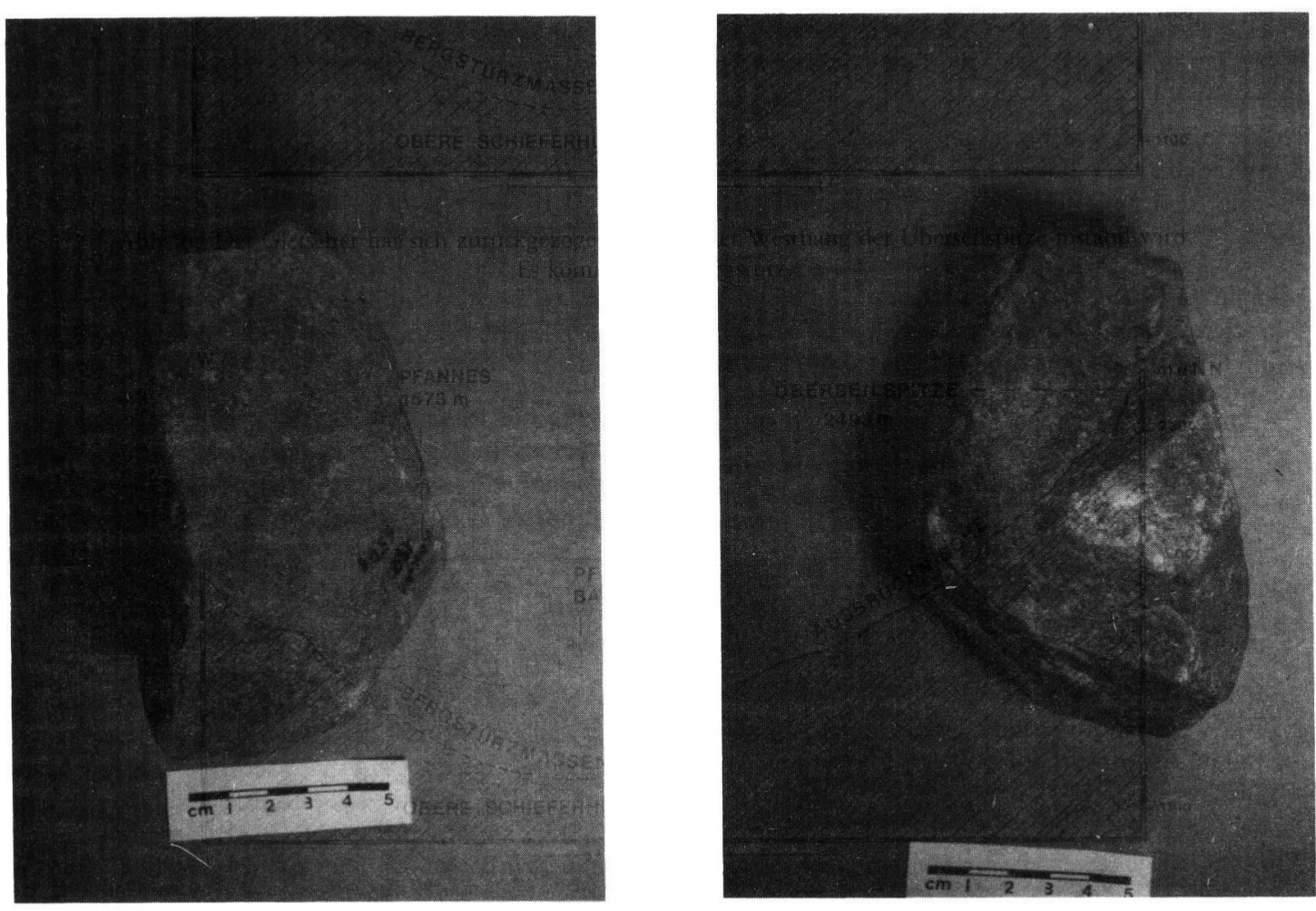


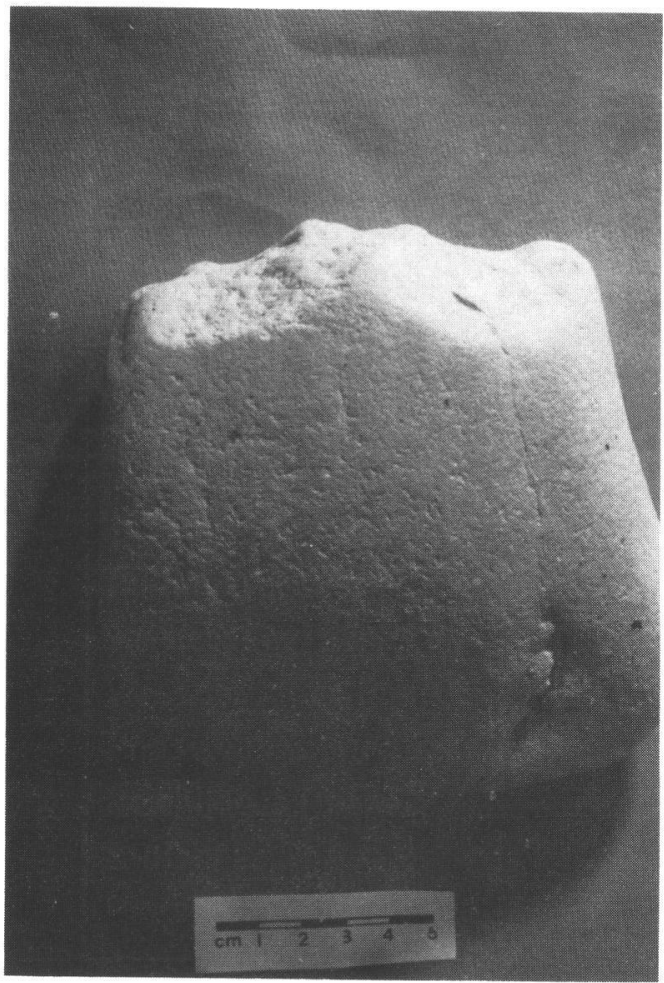

c.

1

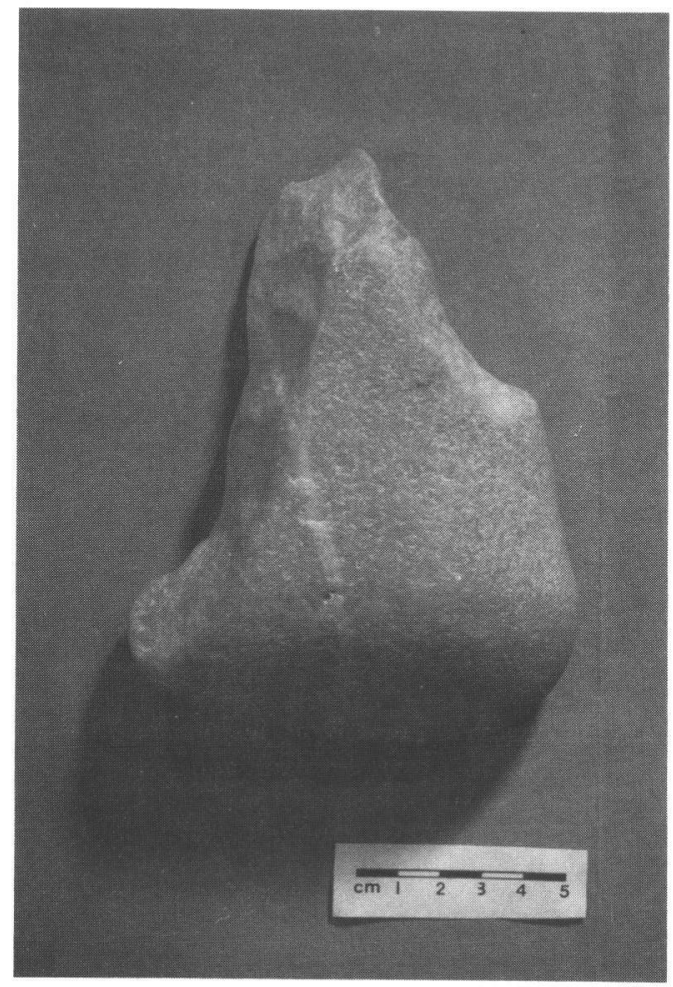

